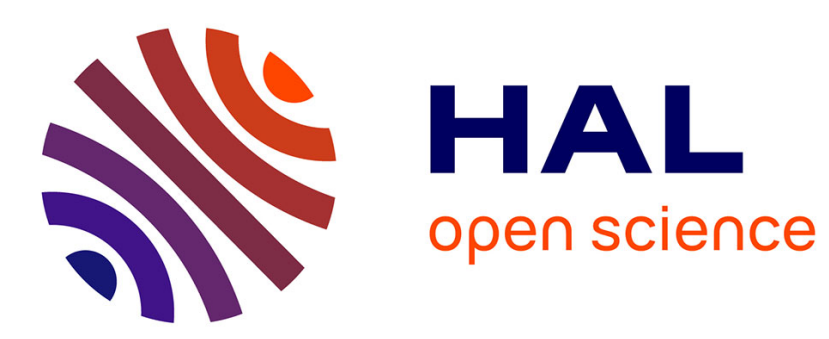

\title{
Les figures du "Juste" et du résistant et l'évolution de la mémoire historique française de l'occupation
}

\author{
Sarah Gensburger
}

\section{To cite this version:}

Sarah Gensburger. Les figures du "Juste" et du résistant et l'évolution de la mémoire historique française de l'occupation. Revue Française de Science Politique, 2002, 52e année (2-3), pp.291-322. 10.3406/rfsp.2002.403712 . halshs-00926318

\section{HAL Id: halshs-00926318 https://shs.hal.science/halshs-00926318}

Submitted on 9 Jan 2014

HAL is a multi-disciplinary open access archive for the deposit and dissemination of scientific research documents, whether they are published or not. The documents may come from teaching and research institutions in France or abroad, or from public or private research centers.
L'archive ouverte pluridisciplinaire HAL, est destinée au dépôt et à la diffusion de documents scientifiques de niveau recherche, publiés ou non, émanant des établissements d'enseignement et de recherche français ou étrangers, des laboratoires publics ou privés. 


\section{Les figures du « Juste » et du résistant et l'évolution de la mémoire historique française de l'occupation}

In: Revue française de science politique, 52e année, n²-3, 2002. pp. 291-322.

Citer ce document / Cite this document :

Gensburger Sarah. Les figures du « Juste » et du résistant et l'évolution de la mémoire historique française de l'occupation. In: Revue française de science politique, 52e année, $n^{\circ} 2-3,2002$. pp. 291-322.

doi : $10.3406 /$ rfsp.2002.403712

http://www.persee.fr/web/revues/home/prescript/article/rfsp_0035-2950_2002_num_52_2_403712 


\section{Résumé}

L'article propose de renouveler l'analyse de la mémoire historique française de l'Occupation. II met en évidence un processus d'institutionnalisation d'une nouvelle figure, celle du Juste parmi les nations devenu progressivement Juste de France. Cette évolution repose notamment sur un rapport complexe entre ce nouveau personnage mémoriel et celui, plus ancien, du Résis-tant. D'une part, cette mise en place s'appuie sur des modalités symboliques proches de celles utilisées pour les résistants. D'autre part, la figure du Juste de France remplit un rôle fonctionnel autrefois exclusivement dévolu à ces derniers. A travers son incorporation s'opère la réécriture d'une rhétorique dichotomique au sein d'un régime mémoriel renouvelé où une France-patrie des droits de l'homme remplace une France-nation et territoire. Ce constat conduit à l'analyse de la portée réconciliatrice d'une telle mémoire historique et de ses modalités. Celle-ci repose notamment sur les imbrications entre individuel et collectif au cœur des représentations dupasse. L'article incite enfin à un retour critique sur les travaux, des tenants du " paradigme de la mémoire stratégique ». Ainsi, à travers un cas empirique concret, l'auteur se livre-t-il à une réflexion conceptuelle et méthodologique sur la notion de mémoire et ses implications. Apparaît avec force la nécessité de dépasser les approches courantes des phéno-mènes de mémoire, souvent trop linéaires et unidimensionnelles.

\section{Abstract}

The article aims to throw new light on the analysis of French historical memory of the occupation. There is the institutionalization of a new figure, that of the righteous among nations becoming progressively the righteous of France. This development is based on complex relations between this new memorial character and the older one of the Resistant. On the one hand, this character is built on symbolic processes similar to those used for the Résistants, and on the other hand, the figure of the righteous of France fulfills a functional role that previously fell only to the latter. It is incor-porated through the rewriting of a dichotomic rhetoric within a renewed memorial regime in which a France - land of Human Rights replaces a France-Nation and Territory. This observa-tion leads to the analysis of the reconciliatory scope of such a historic memory and its methods. It is based in particular on the intertwining between the individual and the group within the repre-sentations of the past. It fosters a critical review of the work of those who defend the «paradigm of strategic memory ». Thus, through a concrete empiric case, the author deals with a conceptual and methodological thinking on the notion of memory and its implications. It is essential to go beyond the common approaches of the phenomena of memory, all too often linear and one-dimensional. 


\title{
LES FIGURES DU JUSTE ET DU RÉSISTANT ET L'ÉVOLUTION DE LA MÉMOIRE HISTORIQUE FRANÇAISE DE L'OCCUPATION
}

\author{
SARAH GENSBURGER
}

$\mathrm{D}$ epuis dix ans, la mémoire française de l'Occupation a connu de nombreuses évolutions. La plus récente est marquée par la loi du 23 mars $2000^{1}$ adoptée par le Parlement français. Celle-ci institue « une journée nationale à la mémoire des victimes des crimes racistes et antisémites de l'État français et d'hommage aux "Justes" de France qui ont recueilli, protégé ou défendu, au péril de leur propre vie et sans aucune contrepartie, une ou plusieurs personnes menacées de génocide. Cette journée est fixée au 16 juillet, date anniversaire de la rafle du Vélodrome d'Hiver à Paris, si ce jour est un dimanche ; sinon elle est reportée au dimanche suivant. Chaque année, à cette date, des cérémonies officielles sont organisées aux niveaux national et départemental, dans des conditions fixées par décret en Conseil d'État ${ }^{2}$.

Cette loi reprend le décret du président de la République, en date du 3 février 1993, qui instaurait « une journée nationale commémorative des persécutions racistes et antisémites commises sous l'autorité de fait dite "gouvernement de l'État français" (1940-1944) ». Elle ajoute, cependant, un « hommage aux "Justes" de France » aux visées commémoratives initiales de cette journée. Il s'agit là de la première apparition, dans les textes officiels de la République française, du terme de « Juste ».

L'État français fait ici référence au titre de «Juste parmi les nations ${ }^{3}$ créé par l'Institut Yad Vashem de Jérusalem pour désigner, et honorer, ces non-Juifs qui ont pris des risques de façon désintéressée pour sauver des Juifs pendant la seconde guerre mondiale ${ }^{4}$. Ce titre est attribué depuis 1963 par une commission ad hoc présidée par le président de la Cour suprême israélienne et sur la foi d'au moins deux témoignages de Juifs sauvés. Son attribution donne lieu à la remise d'un diplôme, d'une médaille

1. Le décret d'application a été pris le 15 juillet 2000 . Le 16 juillet 2000 a donc donné lieu à la première édition de cette journée.

2. Article unique de la loi instaurant « Une journée nationale à la mémoire des victimes des crimes racistes et antisémites de l'État français et d'hommage aux "Justes" de France », adoptée, à l'unanimité, par l'Assemblée nationale, le mardi 29 février 2000 et par le Sénat, le 23 mars 2000.

3. Ce titre a été créé en 1953 dans le cadre de la loi adoptée par la Knesset et créant l'Institut Yad Vashem. Son attribution, au nom de l'État d'Israël, a été confiée en 1963 à un département des Justes parmi les nations, au sein des services de Yad Vashem. Ce titre est attribué dans l'ensemble des pays où des Juifs ont été sauvés. Une commission spéciale examine les dossiers constitués par des personnes sauvées afin d'honorer leurs sauveteurs. 2000 Justes parmi les nations français ont été honorés à ce jour.

4. Ce titre est attribué selon une procédure dont le cœur et l'origine sont constitués par les témoignages de Juifs sauvés. Si, jusqu'à la fin des années 1980 , la constitution des dossiers s'effectuait directement auprès de Yad Vashem Jérusalem, aujourd'hui, et depuis plus de dix ans, le Comité français pour Yad Vashem, association loi 1901, prend en charge le rôle d'intermédiaire entre les témoins et le département des Justes de Jérusalem. Cette association est animée par des Juifs français qui ont, dans leur majorité, effectué eux-mêmes des démarches pour faire honorer ceux qui les ont aidés. 
et à la mention du nom de la personne honorée sur le Mur des Justes situé dans l'Allée des Justes, au sein du site de Yad Vashem ${ }^{1}$.

La reprise législative, par la France, du terme de «Juste » fait donc référence à une figure mémorielle façonnée originellement par un État étranger et située à la croisée de mémoires individuelles, collectives et historiques. De quelle évolution de la mémoire historique française de l'Occupation, cette adaptation nationale est-elle le symptôme ? N'est-elle qu'un aménagement du vocabulaire mémoriel de l'État français ou révèle-telle une profonde restructuration de la mémoire historique française de l'Occupation ? Pour répondre à ces questions, il s'agit de déterminer si cette apparition n'est qu'une manifestation isolée ou si elle s'inscrit dans une évolution continue de cette mémoire. Dans cette perspective, il convient, tout d'abord, de s'interroger sur la signification de la reprise partielle du titre de «Juste parmi les nations » au sein de l'expression de «"Juste" de France ». Cette reformulation linguistique n'est-elle qu'une adaptation nationale d'un titre étranger ou constitue-t-elle la création d'une nouvelle figure nationale ? L'étude des liens éventuels entre résistants, figures mémorielles françaises centrales depuis la fin de la Seconde guerre mondiale, et « Justes » de France est, par exemple, nécessaire pour estimer les éventuelles modifications dont la loi du 23 mars serait l'illustration. En effet, la figure du Résistant constitue le visage officiel traditionnel de la mémoire historique française de l'Occupation. S'interroger sur le rôle éventuel joué par l'évocation des Justes, dans une reformulation de la mémoire historique de l'Occupation, impose donc d'analyser avec précisions les rapports entretenus par les deux figures respectives, du Juste et du Résistant.

Plus largement, si, comme l'explique François Bédarida, «l'enjeu de mémoire qui continue de susciter bien des controverses » est bel et bien « celui de l'attitude des Français à l'égard de la persécution des Juifs $»^{2}$, il importe de comprendre quelle évolution de cet enjeu l'apparition du terme de «Juste » peut signifier.

L'analyse de l'incorporation législative du terme de « Juste » par l'État français peut donc constituer une approche empirique permettant de saisir le fil du renversement opéré en ce qui concerne la mémoire historique de l'Occupation, entendue, au sens de Marie-Claire Lavabre ${ }^{3}$, comme une norme mémorielle officielle, qui tend vers l'homogénéisation des représentations, signifiante pour la société présente et fondée sur les usages sociaux du passé. Enfin, elle est, par là même, l'occasion d'une réflexion théorique sur le concept de mémoire au travers d'un exemple empirique. Plus spécifiquement, la question de la validité de la mémoire en termes purement stratégiques peut notamment constituer l'horizon théorique de cette réflexion ${ }^{4}$.

1. À l'origine, un arbre au nom du Juste était planté dans l'Allée des Justes. Mais suite à une trop grande affluence, cela a dû être abandonné et a été remplacé par ce Mur des Justes.

2. Jean-Pierre Azéma, François Bédarida, «Controverses et enjeux de mémoire », 19381948. Les Années de Tourmente de Munich à Prague. Dictionnaire critique, Paris, Flammarion, 1995, p. 1092.

3. Marie-Claire Lavabre, Le fil rouge. Sociologie de la mémoire communiste, Paris, Presses de Sciences Po, 1994.

4. En effet, si cet article se restreint à cet aspect des phénomènes de mémoire, il ne prétend pas, bien au contraire, qu'une telle étude doive se limiter à cette seule focale. Cet article cherche spécifiquement à mettre en avant un ressort de la mémoire parmi d'autres. II ne veut pas laisser entendre que celui-ci reposerait sur une ou des mémoires vives qui en seraient le support univoque. Il s'inscrit, au contraire, dans un travail de recherche plus large qui se donne pour but d'étudier l'ensemble des manifestations de la mémoire et les interactions complexes qui les réunissent. Ce travail, pris dans son ensemble, s'intéresse, par exemple, aux mémoires individuelles au travers de la conduite d'entretiens non-directifs avec des personnes sauvées qui témoignent. À ce jour, plus d'une quarantaine de 


\section{L'APPARITION D'UN NOUVEAU PERSONNAGE DE LA MÉMOIRE HISTORIQUE FRANÇAISE DE L'OCCUPATION}

La loi du 23 mars ${ }^{1}$ inscrit donc le terme de « Justes » dans un cadre national. Elle donne une place visible et officielle à une figure mémorielle façonnée par un autre État, diverses associations et nombre d'individus. Quel est le sens de cette intronisation ? Résulte-t-elle d'un aménagement rhétorique ou indique-t-elle un changement plus profond ? S'inscrit-elle dans un processus de réappropriation nationale d'une figure mémorielle?

Ainsi, le personnage du «Juste » ne fait-il pas là sa première apparition. Il fut mentionné pour la première fois, dans un discours officiel, le 16 juillet $1995^{2}$, dans la bouche du président de la République. En effet, tous les commentateurs de cette déclaration solennelle ont omis de souligner l'apparition de «"ces Justes parmi les nations" qui, au plus noir de la tourmente, en sauvant au péril de leur vie, comme l'a écrit Serge Klarsfeld, les trois-quarts de la communauté juive résidant en France, ont donné vie à ce qu'elle [la France] a de meilleur ${ }^{3}$.

Cette mention s'est depuis répétée de façon récurrente au plus haut niveau de l'État. Par exemple, deux ans plus tard, dans son discours au Mémorial du martyr juif inconnu, le 5 décembre 1997 , le président évoque à nouveau « l'héroïsme quotidien et discret de tous les «Justes », ces anonymes de toutes conditions, de toutes religions qui sauvèrent, parfois au péril de leur vie, les trois quarts des Juifs de France... ». Ces propos illustrent le recours systématique du président au terme de «Juste » dans ses prises de parole officielles qui ont vocation à nourrir la mémoire historique française de 1'Occupation.

Les figures et les images se situent, structurellement, au cœur des phénomènes de mémoire ${ }^{4}$ et en constituent le langage. Comme l'explique Alfred Grosser, «La mémoire collective est simplificatrice. Elle a besoin de distinguer d'entre les bons et les méchants $»^{5}$, et cette distinction passe par la figure et $1^{\prime}$ image. «Ce n'est pas le passé lui-même qui nous domine, sauf peut-être, par le biais des déterminations biologiques. Ce sont les images du passé. Celles-ci sont, souvent, tout aussi puissamment structurées et contraignantes que les mythes. Images et constructions symboliques du passé se gravent dans notre sensibilité. ${ }^{6}$

Ainsi, « les Justes parmi les nations » des discours sont-ils certes des êtres singuliers mais, avant tout, des personnages, en l'occurrence des héros comme dans les images d'Épinal. L'introduction répétée d'une nouvelle figure au sein du discours

ces entretiens ont été réalisés. Ils ne sont donc pas intégrés au corpus de travail dont ce présent article rend compte. À ce titre, ils doivent être distingués des quelques autres, plus directifs et de type informatif, réalisés auprès de responsables d'institutions ou d'acteurs publics dont les propos, en tant qu'acteurs directs de constitution de la mémoire historique, sont pris en compte dans ce papier.

1. Instaurant «Une journée nationale à la mémoire des victimes des crimes racistes et antisémites de l'État français et d'hommage aux "Justes" » de France, dont l'article unique est cité plus haut.

2. Il y reconnut la responsabilité de la France dans les déportations.

3. Extrait du discours du président de la République, le 16 juillet 1995.

4. Cf. à ce sujet les travaux réunis dans Travail sur la figure, travail de la mémoire, Strasbourg, Éditions de la Maison des Sciences de l'Homme/Centre Marc Bloch, 1997.

5. Alfred Grosser, « La mémoire des peuples », Études, avril 1996, p. 504.

6. George Steiner, Dans le château de Barbe-Bleue. Notes pour une redéfinition de la culture, Paris, Gallimard, 1997, 1'c éd., 1971, p. 13. 
mémoriel officiel sur l'Occupation n'est donc vraisemblablement pas anodine et sa finalité doit être questionnée. Dans quelle mesure indique-t-elle l'existence d'un processus d'institutionnalisation de la figure du Juste ? Toutefois, en 1995, ce personnage mémoriel est encore désigné par le vocable de «Juste parmi les nations ». Il n'est pas encore ce " "Juste" de France » honoré par la loi du 23 mars. Dès lors, si la loi du 23 mars doit être questionnée dans la perspective de ce discours initial, quel sens doit être attribué à cette substitution finale des «"Justes" de France » aux « Justes parmi les nations »?

\section{L'INSTITUTIONNALISATION DES JUSTES PARMI LES NATIONS}

À cet égard, la mise en scène de la cérémonie d'inauguration du « Mémorial en hommage aux Justes de France », à Thonon-les-Bains, le 2 novembre 1997, offre un terrain privilégié pour l'étude de la signification et de l'épaisseur symbolique de l'apparition de ce nouveau personnage, au sein de la mémoire historique française de l'Occupation. En effet, bien qu'elle émane de la communauté juive de France, cette cérémonie se réfère d'emblée aux «Justes de France », contrairement aux cérémonies organisées par le Comité français pour Yad Vashem, qui ont construit la figure du Juste, et concernent, elles, les « Justes parmi les nations ».

\section{RETOUR SUR UNE INAUGURATION}

L'inauguration de ce « Mémorial en hommage aux Justes de France » constitue à ce jour la plus importante manifestation publique concernant les Justes ayant eu lieu dans l'hexagone '. Elle est due à l'initiative du Consistoire central qui, pour ce faire, a créé une association.

La nature « historique » et nationale de la mémoire à laquelle participe ce Mémorial se révèle d'emblée, à travers la participation de nombreuses personnalités de la République française. En effet, la présence au sein de l'Association française pour l'hommage aux Justes parmi les nations d'acteurs du monde politique français constitue une de ses originalités. À l'inverse, le Comité français pour Yad Vashem ne compte, lui, parmi ses membres d'honneur, aucune personnalité choisie en raison de sa visibilité politique et publique ${ }^{2}$. La dimension « historique » et nationale de l'initiative transparaît aussi dans la composition de 1'assemblée présente à l'inauguration.

1. Certes, d'autres manifestations ont et ont eu lieu. Le Comité français pour Yad Vashem organise régulièrement des cérémonies de remises de médailles de «Justes parmi les nations », principalement dans des mairies, dans la capitale et en province. Les plus importantes ont été la cérémonie de remise de médailles des Justes parmi les nations effectuée à l'Assemblée nationale le 2 mai 2000 et l'inauguration de l'Allée des Justes à Paris, le 8 octobre. Cependant, tant la qualité de l'assistance que l'écho médiatique donné à ces cérémonies sont moindres que ceux constatés lors de cette inauguration qui suit de relativement près $(2$ ans) la déclaration de Jacques Chirac du 16 juillet 1995.

2. En effet, Laurent Fabius est président d'honneur de l'Association française pour l'hommage aux Justes parmi les nations. Pierre Mazeaud, Philippe Séguin, Jean Matteoli, président du Conseil économique et social, et Jean Denais, maire de Thonon-les-Bains, en sont également membres. À l'inverse, aucune personnalité non-juive n'est membre du Comité français pour Yad Vashem. 
Cette dernière est placée «sous le haut patronage de Monsieur Jacques Chirac, président de la République ${ }^{1}$. De même, le maire de Strasbourg, alors ministre de la Culture et de la communication et porte-parole du gouvernement, et enfin, le secrétaire d'État chargé des anciens combattants sont mentionnés sur l'invitation à la cérémonie. Sur les sept personnes qui « prient d'assister à la cérémonie d'inauguration », quatre sont des personnalités de la République.

Cette intrusion du monde public au sein d'un hommage censé émaner de la communauté juive de France distingue la constitution du Mémorial de l'expression d'une mémoire collective spécifique aux Juifs de France. Elle indique l'espace social au sein duquel se situe effectivement cette manifestation : la société française tout entière. Il s'agit en fait d'une commémoration « officielle », au niveau de la République française, symbolisée tant par les officiels invités que par la nouvelle «mémoire historique » qu'elle doit nourrir. Ainsi, cet événement est-il un exemple de commémoration telle qu'Annette Wieviorka en décrit 1'évolution : «La commémoration est devenue spectacle. Aujourd'hui, ce qui assure le succès d'une manifestation, ce n'est pas le nombre de personnes qui s'y associent... mais sa couverture médiatique. Aussi faut-il qu'elle fasse événement digne d'être répercuté par les médias et de toucher ainsi un public infiniment plus large que celui qui y participe $\gg^{2}$. L'inauguration d'un tel mémorial est bel et bien un événement dont l'organisation ressemble à une véritable « mise en scène ».

En effet, il s'agit d'une première dont le Consistoire, avec d'autres, a voulu faire un événement médiatique. L'une des deux attachées de presse du Consistoire ${ }^{3}$ mobilisées pour l'événement décrit, d'ailleurs, sa façon de mener l' « opération » en parlant d'《 allumer des projecteurs ${ }^{4}$, comme pour une mise en scène de spectacle. Dès lors, la presse reste un vecteur privilégié. Qu'elle soit nationale ou régionale, écrite ou télévisuelle, elle a largement rendu compte de cette inauguration. Par exemple, « Thononles-Bains : la communauté juive a rendu hommage aux Justes » est le second titre des dépêches de l'Agence France presse du lundi 3 novembre au matin. Les journaux télévisés du dimanche soir ont, tous, diffusé un reportage sur l'événement. De même, la presse écrite 1'a largement couvert. Le Figaro, La Croix, Libération et Le Monde lui ont, par exemple, chacun consacré une page entière.

Cependant, les titres des articles montrent que, si la cérémonie accède au statut d'événement, c'est aussi grâce au contexte général du 2 novembre 1997 . Celui-ci permet notamment de comprendre l'apparition du terme de Juste au sein de la mémoire historique française de l'Occupation et de cerner ce qu'il est supposé figurer. Le 2 novembre 1997, il y a un peu moins d'un mois ${ }^{5}$ que le procès Papon a débuté et l'inauguration du Mémorial est baptisée « opération Ombre et lumière » par le Consistoire.

À la tribune, à Thonon-les-Bains, Mme Trautmann entame son discours par : «Ailleurs, un homme répond de ses actes », tandis qu'à la barre, à Bordeaux, Samuel Pisar ${ }^{6}$ oppose « Thonon » et « Bordeaux ». La cérémonie est donc entièrement conçue

1. Ce dernier est représenté par le préfet de Haute-Savoie, chargé de lire son discours.

2. Annette Wieviorka, «1992. Réflexions sur une commémoration. », Annales, 48, maijuin 1993, p. 708. monie.

3. Rencontrée lors d'un entretien de type informatif réalisé quinze jours après la céré-

4. En effet, 85 journalistes étaient présents.

5. Le 8 octobre 1997.

6. Qui est d'ailleurs président fondateur du Comité français pour Yad Vashem. 
vis-à-vis de Vichy comme la lumière n'existe que par rapport à l'ombre. Néanmoins, cet écho contextuel n'est-il qu'un concours de circonstances ? Ou est-il l'indice de la fonction dévolue à la figure du Juste au sein de la mémoire historique française de l'Occupation?

Le 2 novembre 1997, le président Chirac, par la voix du préfet de Haute-Savoie, fit précisément son deuxième discours officiel concernant Vichy et l'attitude des Français face au sort des Juifs.

En effet, à Thonon-les-Bains, Jacques Chirac reprend l'exacte formulation qu'il a initiée le 16 juillet 1995. Cependant, ce jour-là, Jacques Chirac l'amplifie. Il ajoute : «Mais, si le mal doit être reconnu, le bien ne doit pas être méconnu. Aux heures les plus noires, la noblesse et l'espérance continuaient de vivre. Elles étaient dans le cœur des combattants de la France libre, des résistants, si nombreux dans cette belle province. Dans le cœur, aussi et surtout, de tous ces Français anonymes, ces Justes parmi les nations qui, au plus noir de la tourmente, sauvèrent les trois quarts de la communauté juive résidant sur son sol. »' 1 . Il poursuit en identifiant ces figures singulières que sont les Justes à l'ensemble de la population française de l'époque. Ainsi énumère-t-il des actions de Justes en faisant référence aux diverses catégories de la société française de l'Occupation. Ces catégories sont précisément celles qui, d'un point de vue collectif, ont été les plus accusées et se sont même parfois excusées. Il s'agit des «prêtres », des « Mgr » ${ }^{2}$ et plus largement, de tous ces « chrétiens » ${ }^{3}$, mais aussi des «doyens de faculté » ou des «policiers » et « gendarmes » 4 .

Dans sa conclusion, Jacques Chirac reprend tout le spectre de la société française et identifie globalement les Justes à «l'honneur de la France »: «Ces femmes et ces hommes de toutes conditions, de toutes religions, ces Justes parmi les nations, nous ne les oublierons jamais. Ils sont l'honneur et la fierté de notre pays. Dans une période sombre marquée par la débâcle, les privations, le désarroi moral, ils ont incarné le meilleur de la France : ses valeurs de fraternité, de justice, de tolérance ${ }^{5}$. » ${ }^{6}$

Enfin, au-delà du président Chirac, c'est l'État tout entier qui donne à la figure du Juste sa nouvelle mission au sein de la mémoire historique : celle d'incarner la France authentique. En effet, en raison de la cohabitation, l'exécutif est aussi représenté par le porte-parole du gouvernement, Mme Trautmann. Son discours met en scène la figure du Juste dans une même optique. S'adressant aux Justes, elle leur dit : « C'est

1. Discours de Jacques Chirac prononcé le 2 novembre à Thonon-les-Bains, lu par le préfet de Haute-Savoie. Nous soulignons la formulation centrale qui constitue le cœur de tous les discours présidentiels sur la question et qui, dans une brève formulation, exprime l'ensemble des implications liées à l'institutionnalisation de la figure du Juste. Drancy.

2. La déclaration de repentance des évêques de France a été faite le 30 septembre 1997 à

3. L'écho donné à la visite du pape en Israël et à sa demande de pardon indique l'extrême sensibilité de cette question. Les protestants, eux - notamment à travers le prestige de l'action du village du Chambon-sur-Lignon et de ses pasteurs - sont souvent considérés comme ayant eu une conduite « exemplaire ».

4. Par exemple, l'un des syndicats de policiers s'est excusé au nom de ceux qui avaient participé aux rafles.

5. C'est encore nous qui soulignons.

6. Texte final du discours. 


\section{Les figures du «Juste » dans la mémoire de l'occupation}

sous votre bannière que nous voulons fêter la République... Cette [celle que nous aimons et défendons] France, elle a vos visages, elle a vos mains. » Avec la métaphore corporelle, l'incarnation de la France par la figure du Juste transparaît au sens propre comme au sens figuré. De plus, la présence physique de Catherine Trautmann aux côtés d'une Juste découvrant le Mémorial symbolise le passage du prestige du Juste parmi les nations, figure singulière, à la France tout entière ${ }^{1}$. Cette cérémonie permet donc l'institutionnalisation de la figure du Juste par la République et le passage de témoin entre les Justes et l'ensemble des Français.

\section{JUSTE ET RÉSISTANT : LA COHABITATION DE DEUX FIGURES MÉMORIELLES}

Au terme de ces discours, l'identification officielle est faite entre figure du Juste et la véritable France de l'époque. Le «Juste parmi les nations» est en passe de devenir le «Juste de France ». Il apparaît donc que la mention des «"Justes" de France » au sein de la loi du 23 mars 2000 n'est pas un épiphénomène mais s'inscrit dans la continuité d'un processus précis d'incorporation de la figure du Juste au sein de la mémoire historique française de l'Occupation.

En d'autres termes, au cours de l'opération «Ombre et lumière » de Thonon-lesBains, la figure du Juste devient la nouvelle «part de lumière » de la mémoire historique des « heures les plus noires $»^{2}$. Dans son exposé des motifs, l'auteur de la proposition de loi reprend cette métaphore. «Ombre et lumière de l'Histoire, telle se veut cette proposition de loi qui réunit dans un même texte les deux réalités de notre pays, de notre peuple. $\gg^{3}$

Or, c'est précisément ce terme de lumière qu'Éric Conan et Henry Rousso utilisent pour décrire la fonction traditionnelle de la figure du Résistant au sein de la mémoire historique française de l'Occupation, qualifiée de «part de lumière ${ }^{4}$. $S$ 'agit-il d'un parallélisme rhétorique ou cette métaphore commune reflète-t-elle l'existence de rapports structurels et structurants entre figure du Juste et figure du Résistant? Plus largement, quel (s) rôle (s) jouent les relations entre ces deux figures dans l'évolution de la mémoire historique française de l'Occupation ?

Tout d'abord, l'apparition du terme de Juste au sein des discours mémoriels officiels semble reposer sur un mécanisme complexe de substitution partielle de la figure du Juste à celle du Résistant. Toutefois, ce glissement fonctionnel s'opère grâce à une reprise de la symbolique appartenant à la seconde. En effet, c'est au travers d'une certaine continuité ${ }^{s}$ que le changement de cadre - comme tout changement social - est

1. L'absence de toute personnalité juive aux côtés de ces deux femmes montre une nouvelle fois que le cadre de la cérémonie est celui de la société française tout entière et de sa mémoire historique.

2. Termes de Jacques Chirac, utilisé dans son discours de Thonon-les-Bains.

3. Texte de la proposition de loi instaurant une « Journée nationale à la mémoire des victimes des crimes racistes et antisémites de l'État français et d'hommage aux Justes de France », enregistré à la présidence de l'Assemblée nationale le 22 juin 1999, 1727, p. 5. p. 313.

4. Éric Conan, Henry Rousso, Vichy, un passé qui ne passe pas, Paris, Fayard, 1994,

5. L'analyse de Marc Bloch est à cet égard fondatrice. Cf. Marc Bloch, « Mémoire collective, tradition et coutume. À propos d'un livre récent $\gg$, Revue de synthèse historique, 1925. 


\section{Sarah Gensburger}

possible. L'institutionnalisation de la figure du Juste, dans son rapport avec celle du Résistant, et à ses côtés, semble illustrer l'existence de liens étroits entre « mémoire

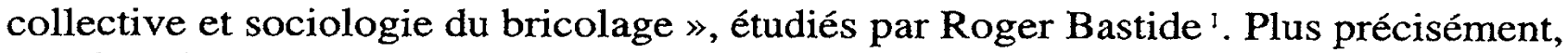
elle fonctionne selon un mécanisme de croisement entre le syntagmatique et le paradigmatique, le fonctionnel et le symbolique. Ainsi, au sein de la figure du Juste, se retrouvent les trois dimensions - matérielle, symbolique et fonctionnelle - propres au lieu de mémoire, telles qu'elles sont évoquées par Pierre Nora ${ }^{2}$. C'est donc par un lien dialectique de différence et de ressemblance avec la figure du Résistant que la figure du Juste se mue en un lieu de mémoire, pilier d'une reconstruction de la mémoire historique française de l'Occupation. Cette dernière consacre l'instauration d'un régime mémoriel renouvelé.

\section{UN CHANGEMENT DE RÉGIME MÉMORIEL}

Dans la tradition gaulliste, la « vraie France » est à Londres et dans les rangs de la Résistance. Ainsi le 14 juillet 1992, alors qu'il répond aux attaques du Comité Vel'd'Hiv' $42^{3}$, est-ce encore cette grille de lecture que François Mitterrand invoque. Il déclare : «Et à cet "État français", on doit demander des comptes, je l'admets naturellement, comment ne l'admettrais-je pas? Je partage totalement le sentiment de ceux qui s'adressent à moi mais, précisément la Résistance, puis le gouvernement de Gaulle, ensuite la Quatrième République et les autres ont été fondés sur le refus de cet "État français", il faut être clair. » Dans un tel cadre, aucun usage n'est fait du terme de Juste. Ce dernier n'a pas de place. Ainsi, en 1994, François Mitterrand refuse-t-il

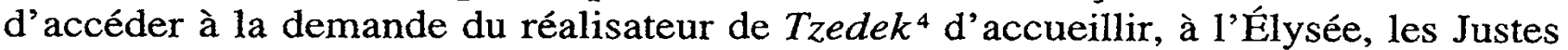
présents dans le film et ne fait aucun discours officiel. S'il accepte finalement de rédiger un texte ${ }^{5}$ destiné à figurer dans le dossier de presse, ce n'est qu'à « la demande expresse du Centre Simon Wiesenthal ${ }^{6}{ }^{6}$. La rhétorique mobilisée par le président de la République le 14 juillet 1992, illustre parfaitement la structure binaire des représentations et la fonction exclusive qu'y occupe la figure du Résistant. Ce discours est une des toutes dernières expressions, au plus haut niveau de l'État, de l'ancien régime de mémoire historique, au sens strict.

En effet, la déclaration solennelle de Jacques Chirac, le 16 juillet 1995, décale ce cadre de la mémoire. En reconnaissant la responsabilité de la France dans les déportations, le président de la République opère un glissement d'une « Francenation », marquée par le patriotisme, accusée de défaite et de soumission à l'ennemi, à une «France-patrie des Droits de l'homme», coupable de crime contre l'huma-

1. Roger Bastide, « Mémoire collective et sociologie du bricolage », Année sociologique, 1970 , p. 65-108.

2. Pierre Nora, Les lieux de mémoire, Paris, Gallimard, 1997, 3 vol.

3. En 1992, celui-ci, constitué de divers intellectuels, a rédigé une pétition demandant la reconnaissance par la République française de la responsabilité de la France dans les rafles et les déportations, notamment celle, hautement symbolique, du 16 juillet 1942 dite « rafle du Vel'd'Hiv'».

4. Nous avons réalisé un entretien de type informatif avec Marek Halter sur l'origine de sa démarche et avons dépouillé tant les brochures de promotion du film que le traitement que la presse en a fait.

5. Cette lettre a été publiée dans la brochure de présentation du film avec la mention : $\ll \mathrm{Ce}$ texte a été écrit à la demande du Centre Simon Wiesenthal ».

6. Élément que le président prend soin de faire connaître au travers de la typographie. 
nité $^{1}$. Pour lui, «La France, patrie des Lumières et des droits de l'homme, terre d'accueil et d'asile, la France, ce jour-là ${ }^{2}$, accomplissait l'irréparable ». Ainsi, reconnaître que la France a une responsabilité dans les rafles et les déportations des Juifs de France, est-ce «évacuer cet acte fondateur » institué par la rhétorique gaulliste. Toutefois, Henry Rousso a raison lorsqu'il soutient, face à Nathalie Heinich ${ }^{3}$, que le président ne modifie pas les structures traditionnelles de la mémoire historique de l'Occupation basées sur une vision dichotomique de la France composée d'une part de Vichy, de l'autre de la Résistance. Ainsi, comme le prévoient Éric Conan et Henry Rousso, « évacuer cet acte fondateur, c'est retrouver, dans un autre contexte, le même dilemme politique, idéologique et moral ${ }^{4}$. Pour ces auteurs, l'expression de ce dilemme ne peut que garder les mêmes traits: «Là réside la contradiction insurmontable : c'est bien le même pays qui a, d'une part, accouché du régime de Vichy et, d'autre part, produit de Gaulle et la Résistance ». Pourtant, ils ne voient pas l'évolution que représente l'introduction du terme de «Juste parmi les nations » comme nouvelle figure de résolution de ce dilemme, aux côtés de la traditionnelle figure du Résistant.

Ce passage d'une France-patrie nationale à une France-patrie des droits de l'homme s'effectue dans un contexte de crise de la figure du Résistant et de redéfinition de ses contours. Au fil des dernières années s'est, en effet, opérée une perte de prestige et de rayonnement symbolique de la figure du Résistant. Ainsi, Jean-Michel Chaumont exprime-t-il une constatation largement partagée lorsqu'il parle de la «marginalisation des mémoires résistantes et antifascistes ${ }^{5}$ et, plus largement, de l'image du Résistant. Les critiques de l'histoire de la Résistance sont d'ailleurs récurrentes ${ }^{6}$. Mais, au-delà de ces polémiques singulières, le délitement du cadre mémoriel établi dans l'après-guerre a conduit peu à peu à se demander : «Que reste-t-il de la Résistance ? ${ }^{7}$, de plus en plus assimilée, à une "supercherie ${ }^{8}$. L'accusation d'indifférence au sort des Juifs souvent portée contre les résistants contribue à cette remise en cause de la figure tutélaire de la mémoire historique française ${ }^{9}$. Plus largement, dans le contexte de la formation d'une mémoire juive de la déportation, la question de l'attitude des Français à l'égard des Juifs connaît « un sort particulier » ${ }^{10}$, comme l'explique François Bédarida dans son Dictionnaire critique. Dès lors, la structure que la Résistance offrait à la mémoire historique française de l'Occupation s'est trouvée, si ce n'est dépassée, du moins affaiblie.

1. C'est un constat du même type que formule Annette Wieviorka : « Alors que jusque dans les années 1960 l'expression couramment employée pour évoquer la période 1940-1945 était "pendant l'Occupation", elle tend à être remplacée depuis les années 1980 par "sous Vichy" " (Déportation et génocide. Entre la mémoire et l'oubli, Paris, Hachette Pluriel, 1995, p. 327).

2. C'est-à-dire, bien sûr, le 16 juillet 1942, jour de la grande rafle du Vel'd'Hiv'.

3. Le débat, 89, mars-avril 1996. Celle-ci déplore notamment la reconnaissance d'une « faute collective » et la « dette » sociale qui l'accompagne.

4. Éric Conan, Henry Rousso, op. cit., p. 454.

5. Jean-Michel Chaumont, «Connaissance ou reconnaissance ? Enjeux du débat sur la singularité de la shoah $\gg$, Le débat, 82, novembre-décembre 1994.

6. Celles formulées contre les époux Aubrac en 1997 ou celles présentant Jean Moulin comme un espion soviétique illustrent cette tendance

7. Titre du numéro spécial de la revue Esprit de janvier 1994.

8. Paul Thibaud, « La République et ses héros. Le gaullisme pendant et après la guerre », Esprit, janvier 1994, p. 64. À tel point que « de Gaulle a fini par n'être que le grand illusionniste (voire le grand menteur) qui a fait croire aux Français qu'ils avaient tous été résistants », p. 64.

9. Là encore nous ne prétendons pas défendre un point de vue plutôt qu'un autre.

10. Jean-Pierre Azéma, François Bédarida, cité. 
Parallèlement, un intérêt certain pour les actes civils ${ }^{1}$ de résistance $s^{\prime} e s t$ fait jour. En effet, l'idée de Résistance est longtemps restée associée à l'image du maquis et de la lutte militaire. Par exemple, en 1994, dans son Livre des Justes, Lucien Lazare déplore encore la «maquisardisation » de la mémoire qui ne « retient que les faits d'armes de la Résistance alors que les actes clandestins de sauvetage $»^{2}$ sont jugés peu dignes de recensement. Pourtant, dès 1989, Jacques Sémelin ${ }^{3}$ attire l'attention académique sur les phénomènes de résistance civile, nombreux dans le corps social. Six ans plus tard, analysant les «images de la Résistance » ${ }^{4}$, François Bédarida constate la naissance d'une nouvelle catégorie d'historiens caractérisée par la «faveur accordée depuis peu à la Résistance civile ${ }^{5}$, cette dernière devenant un nouvel «enjeu de mémoire ${ }^{6}$. Dès lors, la définition de la Résistance est peu à peu reformulée pour se rapprocher de celle donnée, par Lucien Lazare, de la Résistance juive structurée autour du sauvetage ${ }^{7}$. De telles évolutions - qu'il convient de rapprocher d'une diminution, plus générale, de la sphère du politique - ont affaibli la fonctionnalité de la figure du Résistant et ont donc rendu difficile sa transposition intégrale dans une configuration mémorielle renouvelée. Elles ont appelé la redéfinition de ses contours.

FIGURE DU JUSTE ET FIGURE DU RÉSISTANT : UNE MÊME PLACE SYNTAGMATIQUE

Au sein du discours du 16 juillet, la « contradiction insurmontable ${ }^{8}$, la présentation binaire de deux France, perdure donc. Mais la figure du Résistant y occupe une place renouvelée. En effet, en contrepoids à la « faute collective » que Jacques Chirac admet être celle de la France, il évoque ces «Français de Koenig » ou ces « Français libres ». Mais pour incarner « une certaine idée de la France $»^{9}$, la figure des « Justes parmi les nations » rejoint celle du Résistant. Incarnant «l'identité française », les Justes doivent, comme le Résistant, et à sa suite, servir à l'identification de tous les Français, du moins de la grande majorité d'entre eux. Néanmoins, le partage n'est pas strictement équilibré entre les deux figures. Ainsi, seulement deux courtes

1. Cet intérêt pour l'aspect civil des événements n'est pas propre à la vision de la Résistance. Elle touche un ensemble de domaines. Elle marque la fin d'une représentation politique et militaire des conflits. Cette évolution se retrouve, par exemple, dans le développement des actions humanitaires et leur accès à un nouveau statut social, dont l'obtention par Médecins sans frontières du prix Nobel de la paix est l'illustration.

2. Lucien Lazare, Le Livre des Justes, Paris, Hachette Pluriel, 1996.

3. Jacques Sémelin, «Qu'est-ce que résister ? », Esprit, janvier 1994, p. 50-63, et aussi Sans armes face à Hitler, Paris, Payot, 1998 (1 $1^{\mathrm{rc}}$ éd. : 1989).

4. Jean-Pierre Azéma, François Bédarida, cité.

5. Cet intérêt de la recherche historique est synthétisé dans la note critique : Jacques Sémelin, « De la force des faibles : analyse des travaux sur la résistance civile et l'action non violente », Revue française de science politique, 48 (6), décembre 1998, p. 773-782.

6. Jean-Pierre Azéma, François Bédarida, cité, p. 967.

7. Lucien Lazare, La Résistance juive en France, Paris, Le Seuil, 1989.

8. Éric Conan, Henry Rousso, op. cit., p. 454.

9. D'ailleurs, les réactions négatives d'hommes politiques comme Philippe Seguin - pour qui « la France était à Londres » - ou Jack Lang - qui oppose à la conception de Jacques Chirac celle de ses prédécesseurs « qui sont tous des patriotes et de grands résistants »-indiquent que l'évocation des résistants dans ce discours n'a plus ni la même valeur ni la même fonction. Elle sert surtout à lier la nouvelle figure fonctionnelle qui est celle du Juste au cadre mémoriel traditionnel. 


\section{Les figures du «Juste » dans la mémoire de l'occupation}

phrases ${ }^{1}$, au style sobre, presque factuel, sont-elles consacrées à l'évocation de la Résistance alors que les «Justes parmi les nations » sont invoqués dans une phrase au rythme dramatique, autrefois réservé à la seule évocation lyrique de la Résistance.

$\grave{A}$ ce titre, s'il reprend la formulation initiale, ce passage, extrait du discours du président à Thonon-les-Bains, deux ans plus tard, est aussi révélateur de l'apparition de la figure du Juste aux côtés de celle du Résistant : «Aux heures les plus noires, la noblesse et l'espérance continuaient de vivre. Elles étaient dans le cour des combattants de la France libre, des résistants, si nombreux dans cette belle province. Dans le cœur, aussi et surtout, de tous ces Français anonymes, ces Justes parmi les nations. » Par la reprise du mot « cœur » pour évoquer successivement les deux figures, celle du Juste et celle du Résistant, Jacques Chirac les rapproche pour, d'une part, insérer celle du Juste au sein d'une tradition de mémoire historique que la figure du Résistant a longtemps nourri et qu'elle symbolise; pour, d'autre part, réconcilier, via le symbole du Juste, les représentations de la Résistance avec la question du sort des Juifs. Dans l'ordre syntagmatique, un rapprochement progressif s'est donc opéré entre la figure du Résistant et celle du Juste. Cependant, dans la nouvelle configuration de la mémoire historique, un accent est mis sur la figure du Juste. La France n'était-elle pas « dans le cœur, aussi et surtout, de tous ces Français anonymes, ces Justes parmi les nations $»^{2}$ ?

\section{JUSTE ET RÉSISTANT AU PROCÈS PAPON}

Plus encore, la perception différenciée des deux figures mobilisées au cours du procès Papon ${ }^{3}$ fournit une illustration exemplaire de la reconstruction de la mémoire historique française de l'Occupation. En effet, le procès donne à voir le passage de témoin entre figures du Juste et figures du Résistant, au sens propre comme au sens figuré. Ainsi Maurice Papon a-t-il, certes, reçu le soutien de nombreux résistants mais le cœur de la référence n'était pas constituée par le comportement du Résistant. Les témoignages à décharge émanant de résistants n'ont pas provoqué de réel émoi dans la presse ou dans l'opinion, et les décorations pour faits de Résistance détenues par Maurice Papon ont parfois surpris mais pas unanimement choqué. Inversement, lorsqu'il a prétendu avoir sauvé 130 juifs, et a donc voulu revêtir le masque de «Juste », Maurice Papon a été vivement attaqué 4 .

De même, le portrait, et donc aussi et surtout l'«exemplum », proposé, dès le début du procès, par Le Monde, afin de mettre en perspective l'action de Maurice

1. Voici l'expression exacte du Président : « Certes, il y a eu les erreurs commises, il y a les fautes, il y a une faute collective. Mais il y a aussi la France, une certaine idée de la France, droite, généreuse, fidèle à ses traditions, à son génie. Cette France n'a jamais été à Vichy. Elle n'est plus, et depuis longtemps, à Paris. Elle est dans les sables libyens et partout où se battent des Français libres. Elle est à Londres, incarnée par le général de Gaulle. Elle est présente, une et indivisible, dans le cœur de ces Français, ces "Justes parmi les nations" qui, au plus noir de la tourmente, en sauvant au péril de leur vie, comme l'écrit Serge Klarsfeld, les trois-quarts de la communauté juive résidant en France, ont donné vie à ce qu'elle a de meilleur. Les valeurs humanitaires, les valeurs de liberté, de justice, de tolérance qui fondent l'identité française et nous obligent pour l'avenir. »

2. C'est nous qui soulignons.

3. Nous restreignons l'analyse à ses grandes lignes considérées dans notre perspective car les différents témoignages du procès et les registres de justification qu'il a mobilisés mériteraient la réalisation d'une recherche spécifique.

4. Cf., par exemple, Libération du 3 décembre 1997. 
Papon, ne fut pas celui d'un Résistant, mais bien celui d'un Juste parmi les nations, fonctionnaire, qui a justement opéré à Bordeaux : le consul du Portugal, Aristides de Sousa Mendes '. Enfin, lorsque Samuel Pisar ${ }^{2}$ témoigne le 3 mars 1997, quinze jours avant la fin du procès, il cite en exemple, pour fournir une norme de jugement aux jurés, non des résistants, mais ces « trois grands commis de l'État» qui ont été « faits Justes parmi les nations » parce qu'ils ont refusé de faire ce que Papon a fait. Ainsi la figure du Juste at-elle constitué la véritable référence ${ }^{3}$ de ce procès alors que, dans les procès Touvier ou Barbie, nulle mention n'en avait été faite dans le prétoire. Le procès Papon témoigne donc de la mise en place d'une nouvelle mémoire historique dotée de personnages inédits qui se joignent à d'autres pour jouer un rôle de contrepoids.

Alors que l'attitude des Français de l'Occupation envers les Juifs demeure un enjeu de mémoire controversé, la figure du Juste se voit attribuer, au sein d'une mémoire historique reformulée, une fonction importante, auparavant dévolue aux seuls résistants. Désormais, ces derniers, forts d'une tradition de mémoire historique vieille de 50 ans, ne disparaissent pas des discours officiels mais la dimension fonctionnelle de leur évocation est de plus en plus assumée conjointernent avec celle des Justes. Toutefois, au sein d'un processus de bricolage, cette substitution fonctionnelle progressive et partielle s'appuie sur un rapprochement symbolique qui donne à la figure du Juste de nombreux traits de celle du Résistant.

\section{JUSTE ET RÉSISTANT : L'INSTITUTIONNALISATION RÉCIPROQUE DE DEUX FIGURES MÉMORIELLES}

En effet, afin de s'insérer au cœur de la mémoire historique française, la figure du Juste reprend à son compte des éléments du rituel propre à celle du Résistant et des traits caractéristiques des grandes heures de cette dernière.

\section{VALEURS, SYMBOLES ET RITUELS}

Tout d'abord, la figure du Juste est associée aux valeurs morales qui étaient précisément celles des évocations initiales du Résistant. Ainsi, la rhétorique mise en œuvre

1. Le Monde, 31 octobre 1997. D'ailleurs, l'actualité de la figure du Juste et sa signification sur la scène publique française sont une nouvelle fois illustrées par la publication de JoséAlain Fralon, Aristides de Sousa Mendes, le Juste de Bordeaux, Paris, Mollat, 1998. Notons d'ailleurs que le Juste choisi est celui qui a officié à Bordeaux comme Maurice Papon. De même, le numéro spécial sur la shoah de la revue L'Histoire en octobre 1998 a inclu pour la première fois - alors qu'un numéro de ce type sort périodiquement - un article sur les Justes, écrit par Annette Wieviorka, «La gloire des Justes ».

2. Le Monde du 5 mars 1998: «Samuel Pisar rend hommage aux Justes parmi les nations ».

3. Sur ce point, la création du titre par l'Institut Yad Vashem a elle-même une prétention normative. À cet égard, le terme de «Juste » possède un poids symbolique indéniable que le choix de traduction a accentué. En effet, certains linguistes considèrent que « Sage » serait plus proche du terme hébreu de « Hasidé Oummot Ha-olam », à l'origine du titre, qui, dans la Bible, fait référence à ces non-Juifs pieux qui respectent les sept principes qui sont du devoir de toute l'humanité et se montrent bienveillants à l'égard des Juifs. 


\section{Les figures du «Juste » dans la mémoire de l'occupation}

par l'ensemble des acteurs façonnant la figure du Juste identifie-t-elle l'action du Juste à un acte d'agapé ${ }^{1}$, au sens chrétien du terme. Cette rhétorique a été, parfois, appliquée à la Résistance. Par exemple, les propos de Raymond Aron, dans les années 1950, illustrent ce qu'était, symboliquement, la Résistance et comment elle devait être perçue. « La décision de résistance, chez les quelques-uns qui l'ont prise en toute conscience, était de ce type. Elle était décision proprement religieuse, au sens où ce terme dépasse toutes les confessions et toutes les églises... La justification du sacrifice est donnée dans le sacrifice lui-même, comme la justification de la charité dans la charité même, comme la justification de l'amour dans l'amour même. ${ }^{2}$ Acte $d^{2}$ ' amour », voix de la conscience, ce discours sur le « don gratuit » est celui qui est tenu, actuellement ${ }^{3}$, au sujet des Justes. Parallèlement, la crise actuelle de la Résistance conduit à une perte partielle d'écho symbolique à son sujet. Comme la fonctionnalité, cette rhétorique de l'agapé, initialement propre à la figure du Résistant, est réactivée par celle du Juste.

Plus largement, il apparaît que la figure du Juste se trouve institutionnalisée via les canaux et les symboles instaurés originellement pour la seule figure du Résistant. Ce processus de «bricolage » transparaît, par exemple, dans les éléments de rituel utilisés lors de la cérémonie d'inauguration du 2 novembre 1997. Tout d'abord, le lieu choisi, Thonon-les-Bains et, plus largement, la Haute-Savoie, font partie de l'imagerie de la Résistance. En effet, le maquis du plateau des Glières et son martyr figurent, au sein de la mémoire historique construite suite à la seconde guerre mondiale, parmi les faits les plus emblématiques de la Résistance. Et c'est en tant que tel que le lieu fut perçu. Les articles de presse comme les discours indiquent la même imbrication entre les deux figures. Jacques Chirac salue, par exemple, « les combattants de la France Libre... si nombreux dans cette belle province ${ }^{4}$. Ainsi, le lieu ${ }^{5}$, lui-même, permet-il un passage de témoin symbolique entre la figure du Résistant et celle du Juste.

Au-delà du lieu, le déroulement de la cérémonie est, lui aussi, significatif. Tout d'abord, des associations de déportés non exclusivement juives se sont associées à cette initiative ${ }^{6}$. De plus, sur les papiers officiels, personne n'est désigné du seul titre de «Juste parmi les nations». Ce dernier, réduit au seul terme de «Juste», se trouve systématiquement joint à un autre. Par exemple, la Juste à qui revient l'honneur d'inaugurer le Mémorial est décrite, dans la liste des participants au débat intitulé « ceux qui ont osé dire non à Vichy ${ }^{7}$, comme « résistante-juste ». Ce trait d'union

1. Luc Boltanski, L'amour et la justice comme compétences, Paris, Métailié, 1990.

2. Cités par François Bédarida, cité, p. 969.

3. L'étude comportementale sur les Justes qui a eu le plus d'écho a donné lieu à un livre justement intitulé Conscience and Courage (Eva Fogelman, Conscience and Courage : Rescuers of Jews during the Holocaust, New York, Anchor Books, 1994).

4. De même, celui de Catherine Trautmann ou du maire de Thonon-les-Bains qui parle de sa ville comme d'une « terre de fidélité et de résistance ».

5. D'ailleurs, l'intention était à l'origine du choix du lieu. En effet, Paris, qui est pourtant la capitale de cette France qu'on voulait honorer mais aussi le lieu du siège du Consistoire central, n'a pas été choisi. Le choix de Thonon-les-Bains nous fut notamment justifié par l'équipe en charge du dossier au Consistoire, lors d'un entretien informatif, comme correspondant à l'échelle à laquelle celui-ci voulait rayonner «celle de la société française ». La référence n'est donc pas la taille, le poids économique ou autre mais bien la place historique pour la période et donc, son lien avec la Résistance.

6. La Fondation mémoire de la déportation - à travers la présence de sa présidente, Mme M. J. Chombart de Lauwe, au sein du Comité d'honneur -, l'Association mémoire des déportés et résistants d'Europe, par l'intervention de sa présidente au cours de la table ronde, mais aussi Jean Mattéoli, célèbre résistant, en sont quelques exemples.

7. Cette expression fait écho à la présentation traditionnelle de la Résistance. 
est à l'image du rôle joué par la cérémonie dans l'établissement d'un lien symbolique entre les deux figures. Enfin, à côté du Kaddish de Ravel qui rappelle l'origine juive de la démarche, la $27^{\circ}$ division d'infanterie de montagne entonne le chant des partisans, véritable hymne de la Résistance. De plus, des résistants, arborant leurs médailles, se tiennent à côté du bataillon. Ce rituel, hautement symbolique, montre que l'intronisation du Juste parmi les personnages historiques nationaux se fait par rapport, que ce soit pour les différencier ou les rapprocher, à celle du Résistant qui a tant marqué la mémoire historique française.

\section{MISE EN CEUVRE INSTITUTIONNELLE ET ADMINISTRATIVE}

Plus encore, les canaux propres au fonctionnement institutionnel et administratif de la Résistance, sont mobilisés pour faire de la figure du «Juste parmi les nations » celle du « «Juste» de France ». Aux côtés des deux représentants de l'exécutif', l'autre membre du gouvernement présent, le 2 novembre, est précisément le secrétaire d'État chargé des anciens combattants qui a habituellement en charge les résistants. Les Justes deviennent des « anciens combattants », du moins symboliquement ${ }^{2}$, et se rapprochent, dès lors, de ces « combattants volontaires ». Plus concrètement, ce sont les modalités d'institutionnalisation de la Résistance - récompenses, statut des associations, concours de la Résistance et de la déportation ou bien encore célébrations qui sont mobilisées afin de fournir un cadre et une structure privilégiés à l'incarnation mémorielle de la figure du Juste.

En effet, à côté de nombreuses cérémonies locales, plusieurs démarches s'inscrivent dans cette perspective. Ainsi, une liste des Justes fonctionnaires a-t-elle été transmise aux services compétents en vue de l'attribution de la Légion d'honneur. L'État est en passe de reconnaître l'Association des Justes de France ${ }^{3}$ comme étant une association de Résistance et il justifie cette décision en évoquant la présence de nombreux résistants parmi les Justes. Or, qu'il soit avéré ou non, cet état de fait n'est pas survenu soudainement. C'est donc bel et bien la mise en place d'un nouveau cadre de la mémoire historique et la volonté d'incorporation, et donc d'institutionnalisation, qui lui est liée qui motivent l'octroi de ce statut prestigieux, national et républicain, à l'Association.

La Juste parmi les nations qui a inauguré le Mémorial a précisément reçu l'Ordre national du mérite, pour ces deux titres, suite à la cérémonie où elle incarnait « l'honneur de la France ». D'ailleurs, le Mémorial a explicitement été construit afin de générer des structures institutionnelles et administratives identiques à celles qui existent au sujet de la Résistance. Ainsi, aux yeux du Consistoire central, le Mémorial de Thonon-les-Bains se distingue nettement de celui de Yad Vashem. Le premier se situe, lui, dans un contexte national, se rattache à l'histoire de France ${ }^{4}$.

1. Qui sont la porte-parole du gouvernement-ministre de la Culture et le préfet qui représente le président de la République. ce sens.

2. Nous verrons dans la suite de cet article que des mesures concrètes vont être prises dans

3. Association loi 1901 qui a pour but la préservation du souvenir des Justes et de leurs familles. Elle regroupe principalement des Justes mais son adhésion est ouverte.

4. Au cours d'un entretien informatif que nous avons réalisé, l'attaché de presse s'emporte : «C'était pas à Israël de le faire et puis c'est tout. Il y aurait dû avoir un département qui aurait dû s'en occuper en France, comme des résistants. Il y aurait dû avoir quelque chose, une médaille par exemple, qui aurait dû être créée. » 


\section{Les figures du «Juste » dans la mémoire de l'occupation}

De même, les préfets s'enquièrent de plus en plus des Justes résidant dans leur région afin de leur réserver les mêmes égards qu' aux résistants et de les commémorer ${ }^{1}$. Ils organisent des cérémonies à l'occasion du 16 juillet. À Paris, le 8 octobre 2000 , une rue a été rebaptisée «Allée des Justes » dans le quatrième arrondissement de Paris ${ }^{2}$.

Enfin, le Consistoire, en coopération avec l'institution républicaine qu'est la mairie de Thonon-les-Bains, a l'intention d'ouvrir un « Livre de reconnaissance de la communauté juive de France » qui archiverait des actions de sauvetage bien au-delà de celles qui donnent droit à l'attribution du titre de Juste parmi les nations par Yad Vashem. Les personnes mentionnées dans ce recueil vont porter le «titre », plus informel et plus large, de « gardiens de la vie ». Dans la même perspective, le Consistoire a proposé un sujet ${ }^{3}$ sur les Justes pour un prochain Concours national de la résistance et de la déportation et a fait une demande auprès du ministère de l'Éducation nationale pour que le problème des Justes soit un des prochains thèmes. D'une part, il apparaît que le strict titre est peut-être trop restreint pour remplir la fonction attendue de la mise en avant des sauvetages de Juifs par des Français. Les «Justes parmi les nations » ne sont-ils pas officiellement que 1900 alors que, selon l'État français, ils sont censés représenter «l'honneur de la France », dans son ensemble. La figure du Juste est ainsi présentée de façon extensive, englobant les résistants militaires et civils. D'autre part, ce titre « appartient » à l'État d'Israël et non pas à l'État français.

\section{NOUVELLE MÉMOIRE HISTORIQUE ET LIMITES D'UN BRICOLAGE}

L'origine étrangère du titre de « Juste parmi les nations » peut restreindre le processus de bricolage indispensable à une totale institutionnalisation de la figure du Juste au sein de la mémoire historique française de l'Occupation. À ce titre, la loi du 23 mars instaurant une journée commémorative marque autant une nouvelle étape que la prise de conscience de l'existence de limites au bricolage entamé.

JUSTE PARMI LES NATIONS ET COMBATTANT VOLONTAIRE DE LA RÉSISTANCE : DEUX TITRES POURTANT ÉTRANGERS L'UN A L'AUTRE

Les rédactions respectives de la proposition de loi et du rapport de la Commission parlementaire qui en a débattu se situent parfaitement dans la continuité de cette évolution de la mémoire historique organisée autour de la figure du Juste. Pour le rédac-

1. Le Comité français pour Yad Vashem, où nous effectuons une observation participante, reçoit de nombreux courriers et coups de fil en ce sens.

2. Un voyage commémoratif et pédagogique entre Paris et Auschwitz via la clairière des Justes à Thonon a été organisé durant le week-end du $1^{\text {er }}$ mai 2001 , en hommage aux victimes de la déportation et aux Justes. Cette initiative rassemble des associations mais aussi l'État français et, notamment, différents ministères.

3. La proposition de sujet est formulée en ces termes : «"Il y a eu cet immense réseau de braves gens qui ont sauvé tant d'êtres humains!" Robert Badinter. Durant l'Occupation, l'engagement a consisté en aide, assistance et asile à ceux qui étaient recherchés et traqués : résistants, juifs, agents alliés. a) Pour les classes de première et terminale, quelles réflexions vous inspirent ces engagements individuels souvent anonymes; b) pour les classes de troisième, seconde et lycées professionnels : rapportez des actions individuelles ou collectives de votre région ou dont vous avez entendu parler. Dites ce que cela vous inspire ». 


\section{Sarah Gensburger}

teur de la proposition, il s'agit, par exemple, d'une France « des droits de l'homme, de Londres et du Vercors ». Dans ses conclusions, le rapporteur de la Commission désigne les Justes en les situant dans un rapport de différenciation/identification avec les résistants. «Au côté de l'héroïsme de la Résistance, une autre lumière continuait de briller dans la nuit. ${ }^{1}$ Ainsi, parmi les sept institutions reçues par cette Commission chargée de juger de l'opportunité d'un titre français pour les Justes, figure la Fondation de la résistance représentée par M. Mattéoli ${ }^{2}$. Du « don de soi » au « courage », le langage lui-même - comme l'ensemble des discours de la séance du 29 février traduit à nouveau ce rapprochement symbolique fait de différences et de ressemblances. Enfin, c'est au secrétaire d'État aux anciens combattants que revient la tâche de clore les débats et celui-ci termine en rapprochant à nouveau les deux figures : «Merci d'avoir pris l'initiative d'inscrire dans notre calendrier commémoratif national une date qui permette, chaque année, de rendre hommage aux victimes de la politique d'exclusion du gouvernement de l'État français ainsi qu'à ceux des nôtres qui, aux côtés des résistants, ont été les meilleurs d'entre nous. » ${ }^{3}$.

Néanmoins, le titre de Juste parmi les nations ne dépend pas de l'État français. Créateurs du titre, ce dernier et l'Institut Yad Vashem ont, au travers des traits qu'est censée revêtir la figure du Juste, un droit institutionnalisé de jugement sur les comportements individuels. Si la France veut utiliser la figure du Juste dans un contexte qui lui est propre, elle peut vouloir s'écarter des contours institutionnels, relativement stricts, imposés par Yad Vashem. Ainsi, l'intérêt de la France pour la figure du Juste marque-t-il la fin de la répartition des rôles résumée, à travers un exemple concret, par Lucien Lazare. Évoquant un résistant français également fait Juste parmi les nations, celui-ci écrivait, en 1994 : «Alors que le gouvernement français le distinguait pour le sauvetage des Français et des Alliés recherchés par l'ennemi, Yad Vashem lui a décerné le titre de Juste pour le sauvetage des Juifs recherchés ${ }^{4}$. S'il veut dépasser ce fractionnement des prérogatives, l'État français doit, dès lors, se réapproprier la figure du Juste ou, du moins, lui donner une seconde forme, nationale, dont il aurait le contrôle. Tel semble être le préalable à une véritable officialisation de la place de la figure du Juste au sein de la mémoire historique.

\section{UNE TENTATIVE POUR CRÉER UN TITRE DE « JUSTE DE FRANCE »}

Le vote de la loi du 23 mars par l'Assemblée est donc une étape décisive dans le processus d'appropriation de la figure du Juste par la République française. Ainsi les Justes entrent-ils, ce jour-là, dans l'histoire de France. Cependant, ce vote marque aussi les limites d'un tel processus. En effet, la proposition de loi originelle était com-

1. Rapport de Daniel Marcovitch au nom de la commission des Affaires culturelles, familiales et sociales sur la proposition de loi de $M$. Jean le Garrec ( $n^{\circ} 1727$ ) instaurant une « Journée nationale à la mémoire des victimes des crimes racistes et antisémites de l'État français et d'hommage aux Justes de France $»$, document $n^{\circ} 2195$, mis en distribution le 28 février 2000 , p. 4.

2. Il s'agit du Consistoire central israélite de France, du Centre de documentation juive contemporaine, du Comité français pour Yad Vashem, du Comité représentatif des institutions juives de France, du Mouvement confédéral tsigane, de l'Amicale des anciens déportés juifs de France et de la Fondation de la Résistance.

3. Compte rendu de la $1^{\text {re }}$ séance du mardi 29 février 2000 , p. 10.

4. Lucien Lazare, Le Livre des Justes, op. cit., p. 88. 
posée de 5 articles. Pourtant, suite aux travaux du rapporteur, seul le premier est repris en tant qu' article unique. Il était, initialement, question de créer une commission française pour attribuer un titre de "Juste" de France» au sauveteur de Juifs ". Mais comme 1'explique, le 29 février, M. Jean Le Garrec, lors de sa prise de parole : « La proposition initiale, qui a été amendée, prévoyait l'attribution d'un titre de Juste. Cependant, les auditions auxquelles la Commission a procédé ont fait apparaître des réticences sur ce point, liées notamment à un risque de confusion avec le travail accompli par l'Institut Yad Vashem. Nous ne pouvions pas ne pas tenir compte de ces interrogations légitimes. Aussi, modifierons-nous le texte dans un sens plus symbolique. Il appartiendra ensuite à la République de rechercher les moyens d'exprimer sa reconnaissance. $»^{2}$ La volonté française d'utiliser un titre déjà existant s'est donc heurtée à la légitimité acquise par celui qui en fut le créateur, et jusqu'à aujourd'hui, l'unique animateur ${ }^{3}$. Ce dernier est donc parvenu à éviter l'institution d'une jurisprudence qui aurait divergé de la sienne. Il est sorti vainqueur du conflit qui opposait, au travers de deux institutions, deux lectures d'une même figure ${ }^{4}$.

Cependant, issu d'une contrainte, ce « sens plus symbolique » semble donner à l'État français plus de liberté dans ses usages de la figure du Juste. Plusieurs initiatives devront accompagner et suivre cette «symbolique » proposition de loi. Le rapport de la Commission recommande, par exemple, l'attribution de la Légion d'honneur aux Justes lors des cérémonies du 16 juillet et l'organisation de nombreuses cérémonies au niveau local et régional. Il consacre donc un double glissement du « Juste parmi les nations » au «"Juste" de France », d'un titre concret, décerné à l'issue d'une procédure précise, à un terme symbolique, évoqué lors de cérémonies collectives. Ainsi, en juillet 2001, l'Office national des anciens combattants a pris soin de contacter l'ensemble des Justes habitant chaque région afin de les honorer et de les inviter aux cérémonies de commémoration du Vel'd'Hiv.

Enfin, parallèlement, l'État français s'intéresse aussi au titre de Justes parmi les nations existant, tel qu'il est géré par Yad Vashem. Ainsi, le Premier ministre a-t-il remis à l'institut Yad Vashem un chèque d'un million de francs pour contribuer à la rédaction d'un ouvrage recensant les actions des Justes de France, « le livre des Justes parmi les nations de France », tandis que le président de la République a tenu à en rédiger la préface 5 . De même, le maire de Paris et les pouvoirs publics ont décidé de $\mathrm{s}^{\prime}$ associer à la création ${ }^{6} \mathrm{~d}^{\prime}$ un Mémorial de la Shoah et d'y inclure une partie documen-

1. Mettant fin à la répartition des tâches entre Yad Vashem et la République telle que l'a présenté Lucien Lazare

2. Compte rendu de séance, p. 2.

3. Dans sa forme amendée, la proposition de loi fut soutenue et encouragée par les responsables du Comité français pour Yad Vashem. D'ailleurs, le travail d'observation participante que nous avons réalisé lors de notre DEA a montré la place importante, et complexe, tenue par le sentiment d'appartenance de ces bénévoles du Comité Français. Ils ont exprimé, à plusieurs reprises et dans des contextes différents, leur fierté d'avoir vu les Justes, et donc, indirectement eux-mêmes, entrer dans l'Histoire de France.

4. Comme l'illustre cet épisode, nous n'avons aucunement l'intention de nier l'existence de conflits dans le champ de la mémoire. Il est certain que ceux-ci existent. Cependant, dire cela n'apprend rien à l'observateur sur les mécanismes même de la mémoire. Ils sont infiniment plus complexes que la présentation que peuvent en faire les tenants du paradigme de la mémoire stratégique. Derrière des institutions ou des individus qui s'affrontent, peuvent souvent être rangés des points de vue sur la mémoire liés à des positions et des parcours de vie différents au sein des divers groupes. Cf. la fin de l'article.

5. Cf. notamment : France-Israël Information, juillet-août-septembre 1999, p. 8.

6. Dans le cadre de la restructuration du Centre de documentation juive contemporaine. 
taire et commémorative dédiée aux Justes parmi les nations. La rue mitoyenne a été rebaptisée « Allée des Justes » par le cadastre.

La mise en place de ce nouveau visage de la mémoire historique qu'est la figure du Juste prend donc plusieurs formes. Elle rencontre tant des forces de mémoire convergentes que des obstacles qui l'obligent à trouver de nouvelles modalités. Elle montre enfin qu'en termes de mémoire, ce qui apparaît, cristallisé, dans l'espace public n'est finalement que l'écume des choses au sens où les processus ne sont pas linéairement stratégiques. Il résulte de forces de mémoire distinctes à la fois structurées et structurantes ${ }^{1}$.

\section{FIGURES, MÉMOIRE ET RÉCONCILIATION}

Cependant, il ressort de ce bricolage, même limité, que la figure du Juste se trouve institutionnalisée aux côtés de celle du Résistant afin de maintenir un rythme binaire et dichotomique au sein du récit national porté par la mémoire historique française de l'Occupation. Plus largement, l'identification entre « honneur de la France $\gg^{2}$ et figure du Juste, au sein des discours où celle-ci est mobilisée, fournit un cadre à la réconciliation ${ }^{3}$ de la nation avec elle-même. Il est, à cet égard, remarquable que l'Assemblée, le 29 février 2000, comme le Sénat, le 23 mars 2000, ont tous deux adopté le texte à l'unanimité et dans une harmonie parfaite des positions exprimées.

Ainsi, l'ensemble des cérémonies ou commémorations qui sont organisées afin d'honorer les « «Justes » de France » reconstitue-t-il, symboliquement, un espace social commun entre Juifs et non-Juifs au travers d'une assistance composée de ces deux groupes, que les souvenirs des persécutions de Vichy peuvent présenter comme antithétiques. De même, la presse ${ }^{4}$ semble procéder à une solide association entre déculpabilisation et figure du Juste.

Au sein de la mémoire historique, cette figure semble donc constituer un enjeu de mémoire considérable afin de soustraire les non-Juifs au sentiment potentiel de culpabilisation. Selon quel(s) mécanisme(s) ce lien entre figures de Justes individuels et déculpabilisation sociale s'établit-il ? Il n'est, en effet, pas automatique. En Pologne, où pourtant le nombre de Justes ${ }^{5}$ est le plus élevé d'Europe, ce rapport est inversé. À l'opposé, en France, tandis que « seulement»1900 Justes ont été reconnus par Yad

1. L'interaction des divers niveaux de mémoire (individuelle, historique, collective) et des forces qui les portent n'est pas abordée ici car elle suppose de tenir toutes ces focales ensemble. Elle est par contre au cœur de notre travail de recherche sur « la mémoire des Justes parmi les nations en France ». Ainsi, aux analyses de discours et de textes officiels, s'ajoutent la réalisation d'entretiens non-directifs et la conduite de travaux d'observation participante sur plusieurs terrains. Ici, nous avons choisi de nous restreindre au niveau de la mémoire historique française des Justes.

2. Terme employé, à Thonon, par Jacques Chirac, Catherine Trautmann et de nombreuses personnalités, comme des journalistes.

3. Au niveau de la mémoire individuelle, qui n'est pas notre présente focale d'approche, cette réconciliation concerne jusqu'aux différentes identités qui peuvent se rencontrer dans un seul et même individu.

4. Nous avons choisi de nous concentrer sur le traitement par la presse de grands événements où les Justes pouvaient figurer au propre ou au figuré.

5. 5503 au 1.01.2001. 


\section{Les figures du «Juste » dans la mémoire de l'occupation}

Vashem en décembre 2000, il semble que parvienne effectivement à se constituer une politique étatique de réconciliation de la nation avec elle-même.

\section{MÉMOIRE, RÉCONCILIATION ET STATISTIQUES}

Un tel processus, le rapprochement de deux catégories - les non-Juifs et les Juifs - à travers l'évocation d'exemples singuliers - les figures de Justes parmi les nations - pose la question du rapport de l'individuel au collectif au sein de la mémoire ${ }^{1}$. L'effectivité d'un tel mécanisme a déjà été évoquée, par Éric Conan et Henry Rousso, au sujet, là encore, de la figure du Résistant. « En 1940, aucun corps constitué, aucun parti, aucune structure collective n'a appelé à résister... Aucun n'étant sorti indemne de l'épreuve, aucun ne peut échapper au sentiment de culpabilité, sinon en célébrant le souvenir de quelques hommes et de quelques femmes exceptionnels, dont les noms ornent les plaques commémoratives des lieux publics, mais qui, au bout du compte ne représentaient à l'époque qu'eux-mêmes. $»^{2}$ Alors que, «à l'époque », «ils ne représentaient qu'eux-mêmes », une fois réinsérés et mis en scène dans une mémoire du passé, ils sont autant de figures incarnant le groupe auquel ils appartiennent. Précisément, la référence aux divers groupes qui composent la société française est, nous l'avons vu, une structure centrale des discours de Jacques Chirac évoquant la figure du Juste. Ainsi, les associations mentales effectuées entre collectif et individuel, mises en évidence autour de la figure du Juste, sont-elles l'indice de la perméabilité de la frontière entre ces deux registres pourtant a priori antithétiques. D'ailleurs, dans sa «présentation » des Lieux de mémoire 3 , Pierre Nora souligne cette caractéristique centrale des représentations qui ne véhiculent le tout que par le souvenir du détail. «La représentation exclut la fresque, le fragment, le tableau d'ensemble ; elle procède par éclairage ponctuel, multiplication de prélèvements sélectifs, échantillons significatifs $»^{4}$. Le rayonnement de la figure du Juste est une illustration parfaite des propos de l'auteur. Dans toutes les évocations de cette figure, il s'agit donc de mobiliser de façon collective un prestige individuel.

Dans le contexte français, c'est via cette imbrication mémorielle entre collectif et individuel que la figure du Juste est mobilisée au niveau étatique comme un vecteur de réconciliation de la nation avec elle-même et de déculpabilisation du collectif qu'est la société civile française. En fait, au sein de cette dernière, si l'évocation de figures de Justes individuels influence directement et positivement les représentations du comportement collectif, c'est parce qu'elle est jointe à un rappel systématique des

1. C'est d'ailleurs à l'aune de ce couple de notions que Nathalie Heinich, à l'occasion de son dialogue avec Henry Rousso, résume les deux alternatives auxquelles le président Jacques Chirac était confronté lors de son discours de 1995. «On aurait d'une part une éthique du pardon et de la dette, basée sur la culpabilité collective et d'autre part, une éthique de la justice, basée sur la responsabilité individuelle», et «Si l'on reconnaît une culpabilité collective, comme le fait Chirac, le jugement devient impossible: la Justice ne se rend que pour les individus ». Or, si les problèmes de culpabilité sont bien perçus à l'aune du rapport entre individu et collectivité, il semble que la séparation nette, à la fois logique et juridique, à laquelle Nathalie Heinich fait appel, n'ait aucune effectivité au niveau des représentations. Cf. Nathalie Heinich, «Sortir du silence : justice ou pardon ? », Le débat, 89, mars-avril 1996, p. 194 et suiv.

2. Éric Conan, Henry Rousso, op. cit., p. 347. Les auteurs ont souligné.

3. Pierre Nora, op. cit., p. 35.

4. Ibid. 
statistiques. Ce dernier permet l'identification du plus grand nombre, et donc très vite de tous, à la figure du Juste.

En effet, les trois quarts des Juifs de France ont échappé à la déportation ${ }^{1}$. Ce pourcentage est parmi les plus élevés d'Europe. Cette affirmation est une donnée objective et acceptée par tous les historiens ${ }^{2}$, qui n'en donnent pas pour autant d'explication univoque. Toutefois, lorsque la figure du Juste est invoquée au niveau étatique, seul l'aspect statistique est mentionné. Dès lors, l'évocation des « «Justes » de France », au sein d'une mémoire historique française de l'Occupation renouvelée, repose sur un syllogisme : il existe des Français - les «Justes parmi les nations» rebaptisés « "Justes" de France »-qui ont aidé des Juifs ; or, les trois quarts des Juifs de France ont survécu aux rafles ; c'est donc que la majorité des Français ont prêté, le plus souvent anonymement, assistance aux Juifs et les 2000 Justes officiellement reconnus en France n'en sont qu'une infime fraction ${ }^{3}$. Majeure, mineure, conclusion, telles sont donc les trois étapes de ce syllogisme qui rend quasi-automatique, au niveau officiel, l'association entre figure du Juste et déculpabilisation collective de la société civile française.

Ainsi, les discours officiels français ont-ils, bel et bien et avec insistance, systématiquement recours à cette donnée chiffrée qui, à elle seule, donne, par avance, un écho collectif considérable à l'évocation d'un seul de ces Justes. Dans ses trois déclarations sur la déportation, Jacques Chirac fait systématiquement référence aux statistiques de survie des Juifs de France. Il ne mentionne jamais l'effectif de ces 2000 Justes parmi les nations recensés en France à ce jour mais uniquement, et immanquablement, « ces Justes parmi les nations qui ont sauvé les trois-quarts de la communauté juive de France ».

De même, la mention de cette statistique est récurrente dans les textes de préparation de la proposition de loi et dans les débats qui ont précédé son adoption. Ainsi, la proposition initiale ${ }^{4}$ fait-elle directement suivre le rappel de la complicité de l'État français par l'évocation de ces « milliers » de Français qui ont sauvé « les trois quarts de la communauté juive de France, comme l'écrit Serge Klarsfeld $»^{5}$. Le rapport de la Commission qui aboutit au texte définitif ${ }^{6}$ adopte une construction identique, louant

1. Le terme « échappé » ne doit pas, ici, être confondu avec la notion de sauvetage. Il est objectivement établi que des Juifs de France ont échappé à la déportation. Il est différent de dire que cela est dû à l'action de sauvetage des Français. Cf. note suivante $\mathbf{n}^{\circ} 3$.

2. Parmi beaucoup d'autres: Raul Hilberg, La destruction des juifs d'Europe, Paris, Fayard, 1988 ; Annette Wieviorka, Déportation et génocide..., op. cit.

3. Notre intention n'est pas d'établir - ni même de rechercher - la vérité historique sur le degré de représentativité des Justes pour une telle visée mais de déterminer les mécanismes de mémoire qui mobilisent cette idée. Ainsi, notre perspective de recherche est-elle identique à celle adoptée par Annette Wieviorka lorsqu'elle analysait le déroulement de la mémoire du génocide et de la déportation en France et précisait : " Nous n'écrivons pas ici l'histoire des camps de concentration, mais nous cherchons à établir la représentation qui en est donnée dans les années d'après-guerre. » (Annette Wieviorka, op. cit. p. 234). Nous n'entendons pas rendre compte des comportements au sein de la population française sous Vichy, et encore moins de les juger, mais mettre en évidence les représentations qui en sont données et qui façonnent une nouvelle mémoire historique.

4. Proposition de loi, $\mathrm{n}^{\circ} 1727$; op. cit., p. 7.

5. Cette présentation des faits relance d'ailleurs le débat sur le régime de Vichy afin de savoir s'il a permis ou non une plus grande aide de la population civile aux Juifs. Cf. notamment Annie Kriegel, Ce que j'ai cru comprendre, Paris, Robert Laffont, 1991, p. 172, et les développements d'Annette Wieviorka, Déportation et génocide..., op. cit., p. 336.

6. Document $\mathrm{n}^{\circ} 2195$, op. cit., p. 4. 
ces « milliers » de Français qui « ont permis à notre pays de sauver le plus de déportés potentiels du continent européen ». Puis, il ajoute, plus loin : « De ce fait [l'action des Justes], sur les 300000 Juifs que comptait la France en 1939, les trois quarts furent sauvés et échappèrent à la déportation. ${ }^{1} \gg$ Enfin, parmi les interventions des 10 parlementaires ${ }^{2}$, le jour de l'adoption du texte par l'Assemblée, quatre rappellent les statistiques de survie, devenues celles du sauvetage. Presque tous les discours parlent, à propos des Justes, de ces «milliers » de Français alors qu'officiellement, leur nombre atteint à peine deux «milliers ». Enfin, les différents articles de presse qui se placent dans un contexte français rappellent également les statistiques, soit directement, soit au travers des propos du président de la République.

Cette donnée statistique ${ }^{3}$ est donc un ressort fondamental de la mise en place du rapport entre individuel et collectif exigé par l'adoption de la figure du Juste comme nouveau visage de la mémoire historique de l'Occupation. Cependant, si le mécanisme structurant repose toujours sur le lien qui est fait entre l'action honorable de quelques-uns et la déculpabilisation de la majorité, il a pris une nouvelle forme.

\section{UNE FIGURE ENTRE INDIVIDUEL ET COLLECTIF}

En effet, la démarche législative de la République française a, tout d'abord, cherché à établir une procédure similaire à celle de l'Institut Yad Vashem, c'est à dire un titre et une médaille nominatifs. Or, cette dernière se cantonne strictement au niveau individuel. Ainsi, pour Yad Vashem, s'agit-il, à partir de demandes de Juifs sauvés, de distinguer un ou plusieurs individus qui ne valent que pour eux-mêmes. L'examen de chaque dossier par la Commission donne lieu à une nomination individuelle. La notion de réseau n'a, par exemple, pas de sens ici. L'Institut Yad Vashem se refuse d'ailleurs à honorer collectivement du titre de Juste ${ }^{4}$. Le fameux village du Chambon-sur-Lignon n'a, par exemple, jamais reçu de titre de «village Juste parmi les nations $\gg^{5}$ mais, seulement, un parchemin qui, tout en ayant un statut symbolique, ne constitue pas un titre ${ }^{6}$.

1. Ibid., p. 10.

2. Compte rendu de la séance du mardi 29 février 2000 à l'Assemblée nationale.

3. Lorsque les statistiques de survie lors de la Shoah sont analysées, elles donnent lieu à l'expression de jugements moraux. Par exemple, Paul Ricœur juge qu'il faudrait, s'il venait à en effectuer un, «s'attaquer au fantasme d'une pesée, d'un bilan entre la bonté et le crime » («Hommage aux "Justes" », Le Monde, 8 décembre 1994). À l'inverse, Jean Améry considère qu'on ne peut pas s'empêcher d'y penser et que cela revêt une certaine signification (Par delà le crime et le chatiment. Essai pour surmonter l'insurmontable, Arles, Actes Sud, 1995. Publication originelle en 1966, en allemand). Notre mention des statistiques ne se situe pas dans un tel débat mais se contente de restituer l'usage qui en est effectivement fait au niveau de la formulation d'une mémoire historique française de l'Occupation.

4. Ce rapport univoque à l'individuel a pu être analysé et établi grâce à un travail d'observation participante au Comité Français pour Yad Vashem. Par exemple, le Comité français renvoie systématiquement les demandes de titre pour des villages ou des institutions et incite les demandeurs à reformuler leurs requêtes en distinguant des individus au rôle majeur.

5. Titre qui, s'il existait, ferait écho à l'expression de «Village hérö̈que du Chambon-surLignon ». Cf. plus loin.

6. L'action de Yad Vashem n'est pas le thème de cet article. Nous n'en mentionnons donc que les aspects en interaction avec celle de l'État français. Pour plus de détails, cf. Sarah Gensburger, «Le cadre institutionnel de la "mémoire des Justes" », mémoire cité. 


\section{Sarah Gensburger}

À l'inverse, dans le contexte plus général d'une reformulation de la mémoire historique française, le rapport entre individuel et collectif qu'alimente cette proposition de loi est tout à fait différent. La finalité de la reconnaissance républicaine des Justes étant d'emblée collective, la frontière entre groupe et individu est conçue comme perméable. Aussi est-il particulièrement significatif que, lors des travaux et débats suscités par la proposition de loi, le « village du Chambon-sur-Lignon » soit cité à plusieurs reprises par les députés comme un parfait exemple de ce que l'on cherche à désigner du titre de «Juste de France ». Lorsqu'il prend la parole le 29 février 2000, François Baroin illustre cette grille de lecture française de la figure du Juste. Il rappelle les exactions du régime de Vichy, puis poursuit : «Mais la France n'était pas tout entière dans ceux qui trahissaient son esprit, son histoire et son message. Les Français libres, les résistants continuaient le combat par les armes. Partout en France... des Justes sauvaient la vie de Juifs et de tous les persécutés. C'est le village hérö̈que du Chambon-sur-Lignon, ce sont là des prêtres... ${ }^{l}{ }^{1}$ Cette divergence d'interprétation de la relation entre figure du Juste, individuel et collectif, a donc constitué le premier point conduisant à une reformulation de la proposition de loi $^{2}$, en article unique adopté à l'unanimité.

Corrélativement, c'est d'ailleurs à ce niveau que la responsabilité de la France est exprimée par la proposition de loi. Ainsi, dans son rapport qui aboutit à sa reformulation, Daniel Marcovitch précise : « Le droit pénal ne reconnât pas traditionnellement de responsabilité collective... Il importe par conséquent qu'un organe politique, le Parlement, établisse cette responsabilité collective de l'État français, qui a bien été complice de crime contre l'humanité selon la définition de Nuremberg. $\gg^{3}$

Dès lors, le mécanisme se complexifie et l'hommage rendu par la République française à ces figures individuelles que sont les Justes se fera principalement au niveau collectif. Plus explicitement, le premier projet ayant été abandonné, le rapporteur s'en justifie en tentant de construire un autre rapport au collectif. Les actions de sauvetage sont bien individuelles mais elles rachètent la collectivité, elles ne valent donc jamais uniquement par elle-même. La syntaxe et le vocable eux-mêmes traduisent cette idée : «Il faut leur rendre collectivement hommage pour leurs actions individuelles exemplaires, qui doivent guider les générations futures » ou encore : «Plutôt que de leur rendre un hommage individuel, qu'ils ne cherchent pas et qui interviendrait trop tard pour la plupart d'entre eux déjà décédés, il est préférable de se souvenir de leurs actions de manière collective. La force pédagogique de la mémoire en sera d'autant plus grande. ${ }^{4}$ Puis l'un des députés ajoute : "Cet hommage sera d'autant plus fort qu'il aura une portée collective qui rend témoignage de la somme des actions hérö̈ques de chacun. ${ }^{5}$ Le lien entre individuel et collectif est tel que les

1. Compte rendu, p. 8. C'est nous qui soulignons.

2. De plus, un second sujet de désaccord entre l'Assemblée et Yad Vashem a concerné la reprise du titre créé par le second. Ainsi, étant donnée la forme quasi-judiciaire de l'étude des dossiers par les commissions, le risque d'une divergence de jurisprudence a-t-il été soulevé. En effet, cette nature judiciaire se retrouve à la fois dans la Commission des juges de Yad Vashem et dans celle que la proposition de loi voulait instituer. La première est présidée par le président de la Cour suprême israélienne. La seconde devait compter parmi ses quatre membres permanents le président de la Cour de cassation. Enfin, plus largement, étant donné que l'Institut Yad Vashem s'occupe de ces questions depuis 1963, il a considéré que ce titre est indissociable de sa propre activité.

3. Op. cit., p. 5.

4. Propos du rapporteur, op. cit., p. 10 et p. 12.

5. Compte-rendu de la séance, p. 4, propos de Michel Herbillon. C'est nous qui soulignons. 
« Justes villages » se multiplient dans les propos du rapporteur pour y occuper la première place. «On peut aussi parler de « Justes » s'agissant de communautés tels ces villages dans la Creuse, Saint-Pierre-de-Fursac (avec le château de Chabannes), Grand-Bourg ou de la commune Le Chambon-sur-Lignon en Haute-Loire, qui ont collectivement servi de refuge pour des Juifs, particulièrement plusieurs centaines d'enfants qui furent hébergés, cachés et ainsi sauvés de la barbarie. Il faut reconnaître cette solidarité et cette fraternité collectives. ${ }^{1}$ Même s'il ne devient pas titre, le terme de Juste est conservé. Toutefois, il ne l'est que réinterprété à l'aune du collectif afin de permettre un impact social plus large. Ce changement de dimension s'incarne dans le glissement définitif de l'expression de «Juste parmi les nations » à celle de «"Juste" de France », le passage d'une formule individuelle et universelle à un vocable collectif et national.

Le texte définitif de la proposition de loi et les débats qui ont accompagné son adoption illustrent cette lecture du terme de «Juste » lorsqu'ils prétendent en donner l'origine biblique. En effet, pour son rapporteur - comme pour son initiateur - le terme de Juste fait référence à «Celui qui par son action, est capable de sauver le reste de 1'humanité. Souvenons-nous de la parole de Dieu devant Sodome et Gomorrhe : " $\mathrm{S}$ 'il est cinquante Justes dans cette ville, je la sauverai". » ${ }^{2}$ Ailleurs, durant la séance, le thème des 36 Justes du Talmud, dont l'existence sauve l'humanité, est également mentionné ${ }^{3}$. Dans les deux cas, il s'agit du terme hébreu de «tsaddiq ». Il correspond au sens que veut donner la République au terme de Juste en impliquant, par avance, la nature et l'impact collectifs de l'hommage rendu à ces figures individuelles. Or, «tsaddiq » n'est pas ce que traduit l'expression officielle de «Juste parmi les nations », équivalent français de «Hasidé Oummot Ha-olam ». Terme d'origine rabbinique, ce dernier désigne les non-Juifs qui font preuve de bienveillance à l'égard des Juifs sans inclure l'idée d'un lien avec un pardon divin pour la collectivité. «Hasidé Oummot Ha-olam » trouve son sens dans un rapport exclusif d'individus à individus. Si les deux termes sont traduits de façon presque identique, ils n'impliquent donc pas la même relation symbolique entre individuel et collectif. L'association entre le «Juste parmi les nations» et le «Tsaddiq » effectuée par l'Assemblée nationale française est la manifestation, dans le domaine linguistique, de l'existence d'un lien entre individus et collectivités et de sa situation au cœur de la reformulation de la mémoire historique de l'Occupation. La substitution des « Justes de France » aux «Justes parmi les nations » fournit ainsi un cadre à la réconciliation de la nation avec elle-même.

De plus, et simultanément, l'ensemble des actions officielles entreprises par l'État français permet aussi le rapprochement des deux mémoires de la déportation dont la coexistence au sein de la mémoire historique est parfois présentée comme conflictuelle $^{4}$. La relation de renforcement mutuel qui lie figures du Juste et du Résistant permet donc une réconciliation des deux grandes mémoires collectives de la guerre. Le renouvellement de la mémoire historique française de l'Occupation favorise des échanges institutionnels entre des associations ou organisations appartenant à différents milieux de mémoire. Par exemple, la préparation de la loi du 23 mars 2000 a fait dialoguer des représentants de chacune de ces mémoires. Aux côtés des repré-

1. Rapport, p. 9.

2. Compte-rendu de séance, discours du rapporteur, p.1.

3. Ibid., p. 8. Intervention de F. Baroin.

4. Annette Wieviorka, Déportation et génocide..., op. cit. 


\section{Sarah Gensburger}

sentants des institutions juives ${ }^{1}$ figuraient $\mathrm{M}$. Mattéoli, en tant que représentant de la Fondation de la Résistance, et $M$. Lorier, du Mouvement confédéral tsigane. De même, lors des cérémonies du 16 juillet 2001, des associations de Résistance ont accompagné les associations juives.

\section{MÉMOIRE, STRATÉGIE ET ABUS}

La figure du Juste est donc insérée au sein d'une mémoire historique de l'Occupation renouvelée. Elle fournit le cadre de la réconciliation de la nation avec ellemême. Dès lors, des acteurs et des causes doivent-ils être linéairement induits du constat de cette apparition de la figure du Juste ? S'agit-il d' « instrumentalisation », d'《utilisation calculée », voire de «stratégie délibérée », de «manipulation» ou encore d' «abus » de la mémoire historique, qui sont autant de termes employés par les tenants de ce que nous appelons le «paradigme de la mémoire stratégique »? En effet, depuis quelques années, les travaux sur la mémoire développent des analyses en termes d'utilisation stratégique de l'évocation du passé et de concurrence des victimes. La mémoire y est conçue comme un outil aux mains d'acteurs collectifs qui y ont recours et en abusent afin d'obtenir un profit symbolique. Au-delà du cas empirique qu'elle constitue, l'étude de l'institutionnalisation de la figure du Juste dans la mémoire historique française de l'Occupation appelle donc une réflexion conceptuelle et méthodologique sur la notion même de mémoire et ses implications.

\section{LES CONCLUSIONS DU PARADIGME DE LA MÉMOIRE STRATÉGIQUE}

En France, la préface de Stéphane Courtois au Livre noir du communisme: crimes, terreur et répression ${ }^{2}$, La concurrence des victimes, génocide, identité, reconnaissance ${ }^{3}$ de Jean-Michel Chaumont, comme La mémoire, l'histoire, l'oubli de Paul Ricœur ${ }^{4}$ ont, entre autres, repris la critique des Abus de la mémoire 5 faite par Tzvetan Todorov. Ainsi, les développements de ces différents auteurs et l'usage commun qu'ils font du concept d'abus de la mémoire conduisent-ils à l'élaboration d'une approche commune, d'un paradigme de la mémoire stratégique ${ }^{6}$.

Parce qu'il prétend étudier un objet spécifique dans le cadre d'une thèse de sociologie, Jean-Michel Chaumont en fournit le développement le plus abouti. Il associe,

1. Il convient d'ailleurs de noter que contrairement à ceux qui parlent de l'utilisation stratégique de la mémoire par « les Juifs », celles-ci n'étaient pas d'accord sur une position commune. Nous y reviendrons.

2. Livre noir du communisme : crimes, terreur et répression, Paris, Robert Laffont, 1997.

3. Jean-Michel Chaumont, La concurrence des victimes, génocide, identité, reconnaissance, Paris, La Découverte, 1997.

4. Paul Ricœur, La mémoire, l'histoire, l'oubli, Paris, Le Seuil, 2000.

5. Tzvetan Todorov, Abus de la mémoire, Paris, Arléa, 1995.

6. Motivé par la parenté évidente de leurs propos respectifs, le regroupement de ces trois auteurs en un paradigme commun se justifie d'autant plus que Tzvetan Todorov lui-même associe Stéphane Courtois et Jean-Michel Chaumont à son article-réponse à des critiques qui Iui étaient adressées : « Je conspire, Hannah Arendt conspirait, Raymond Aron aussi... », Le Monde, 31 janvier 1998. «Le paradigme de la mémoire stratégique »n'est pas une spécificité hexagonale mais la présente étude se limite au cadre de la société française. 


\section{Les figures du «Juste » dans la mémoire de l'occupation}

notamment, l'utilisation stratégique de la mémoire - qu'il décrit et dénonce - à un renversement du régime d'évocation du passé. Selon lui, au «temps de la honte » se substitue, en 1967 et progressivement, le « temps de la gloire ». Ce renversement serait lié à une hostilité et une concurrence ouverte entre ces deux temps du souvenir devenus deux mémoires distinctes de la Seconde guerre mondiale. Le premier se caractérisait par la «mise en avant» de la figure du Résistant et des valeurs de «courage » et d' « héroïsme ». Le second, lui, s'opère à travers la « glorification » des « Juifs » et la sacralisation de la «souffrance » et du «statut de victime ».

Plus largement, dans son ouvrage, Jean-Michel Chaumont met en évidence une lutte et une hostilité entre les catégories que cette mémoire met en scène, les Juifs et les non-Juifs. Ainsi, si le «temps de la gloire » était porteur de la réconciliation de la nation avec elle-même, le «temps de la honte » recherche la mise en accusation de certains, les non-Juifs, par d'autres, les Juifs, qui font montre d'un souci de «culpabilisation forcenée » 1 des premiers. La seconde période est «entièrement placée sous le signe de l'hostilité des non-Juifs $»^{2}$. Dans le langage de l'auteur, les Juifs ont fini par ne plus exister qu'à travers une souffrance dont les non-Juifs sont les responsables. Ils mettent en avant « la distinction éthico-religieuse - dans le bienentre les Juifs et le reste des hommes $»^{3}$. «Le lien social rompu et corrompu par la guerre entre les Juifs et les gentils $\gg^{4} l^{\prime}$ 'est de façon définitive.

Pourtant, l'analyse de l'institutionnalisation de la figure du Juste au sein de la mémoire historique française de l'Occupation conduit à un constat opposé, presque terme à terme, à celui découlant des analyses du paradigme de la mémoire stratégique. Certes, il est apparu qu'à l'origine de la constitution d'un nouveau visage de la mémoire historique, se situe notamment le reproche fait aux résistants de ne pas s'être intéressés au sort des Juifs. Toutefois, la figure du Juste, sa mise en avant et la relation complexe qui la lie à celle du Résistant semblent être une modalité de renversement totalement différente de celles évoquées par Jean-Michel Chaumont. Bien sûr, la souffrance et l'injustice qui frappèrent les Juifs sont amplement reconnues. Mais elles ne sont pas utilisées, au niveau officiel, afin de stigmatiser et de culpabiliser les non-Juifs de l'époque ou d'aujourd'hui car c'est face à cette injustice que des « héros » ${ }^{5}$ nonJuifs, les Justes, s'élevèrent. Les deux registres, celui de la souffrance et celui de $l^{\prime}$ héroïsme, que Jean-Michel Chaumont ${ }^{6}$ décrit comme étant en concurrence stratégique, sont donc tous deux réunis à travers la figure du Juste. À cet égard, Jean-Michel Chaumont ne tient pas compte des évolutions récentes quand il considère que « nous

1. Jean-Michel Chaumont, La concurrence des victimes..., op. cit., p. 318.

2. Ibid., p. 145

3. Ibid., p. 117.

4. Ibid., p. 232.

5. Il permet également de rétablir l'existence d'un héroïsme des juifs eux-mêmes, question qui fut un enjeu central du premier moment mémoriel. En effet, les actes des Justes supposent d'abord une volonté de se cacher des juifs qui ne sont donc pas ces « moutons » si souvent montrés du doigt. Mais plus encore, l'aide des Justes était souvent apportée en coopération avec des réseaux de résistance juive. La mise en avant de la première permet donc d'insister également sur le rôle des seconds. Nous ne développons pas ce point plus avant ici car il n'est pas au cœur de cette réconciliation mémorielle liée à la reconstruction par l'État de la mémoire historique autour de la figure du Juste. Cependant, encore une fois, elle en résulte en partie au travers d'un jeu de forces de mémoire. Cf. à ce sujet Sarah Gensburger, « Le cadre institutionnel de la "mémoire des Justes" : figures, témoignages et souvenirs », mémoire de DEA sous la direction de Marie-Claire Lavabre à l'EHESS.

6. Et Tzvetan Todorov, op. cit. 
peinons à reconnaître l'héroïsme lorsqu'il n'adopte pas la forme armée »'. En effet, les discours publics montrent parfaitement qu'à travers la figure du Juste, la « configuration héroïque » ${ }^{2}$, mise en place avec la mémoire de la Résistance et dont parlent Éric Conan et Henry Rousso, retrouve un nouveau souffle.

De même, et corrélativement, il est apparu que l'institutionnalisation de la figure du Juste porte une volonté de réconciliation de la nation avec elle-même. Elle nourrit la reconstitution d'un lien social entre Juifs et non-Juifs au sein de la société française, lien qui ne repose pas sur une relation de culpabilisation. L'analyse de l'apparition d'un nouveau visage de la mémoire historique de l'Occupation met donc clairement en question les conclusions des analyses des tenants du paradigme de la mémoire stratégique. Ce constat signifie-t-il pour autant que nous sommes en présence d'une autre «manipulation » ou "stratégie » d'utilisation de la mémoire aux fins opposées à celles mises en avant par les analyses du paradigme de la mémoire stratégique ? Ou signifie-t-il au contraire que la perspective linéairement stratégique en elle-même doit être reconsidérée?

\section{LA MÉTHODE ET LES CONCEPTS DU PARADIGME DE LA MÉMOIRE STRATÉGIQUE : LE PARADOXE ENTRE ABUS ET EXEMPLARITÉ DE LA MÉMOIRE}

La réflexion sur le concept d' « abus de la mémoire », central chez les tenants du paradigme de la mémoire stratégique, semble indiquer que c'est à la seconde question qu'une réponse positive doit être apportée. Elle montre en effet que la faiblesse de ses conclusions provient de celle des concepts, méthodes et outils qu'il met en œuvre. Par la mise en perspective de ces analyses avec celle de l'institutionnalisation de la figure du Juste, il ne s'agit donc pas de fonder des conclusions opposées mais de révéler les lacunes conceptuelles dont les précédentes découlent.

« Disons-le d'un mot, l'exercice de la mémoire, c'est son usage ; or l'us comporte la possibilité de l'abus. Entre us et abus se glisse le spectre de la mauvaise "mimétique". C'est par le biais de l'abus que la visée véritative de la mémoire est massivement menacée. ${ }^{3}$ Paul Ricour, développant le concept initialement élaboré par Tzvetan Todorov, se livre à une analyse des modalités d'exercice des abus de la mémoire. Il les décrit comme, «au sens fort du terme, résultant d'une manipulation concertée de la mémoire et de l'oubli par les détenteurs de pouvoir. Je parlerai alors moins de mémoire blessée que de mémoire instrumentalisée... ${ }^{4}$ C'est à ce plan qu'on peut le plus légitimement parler d'abus de mémoire, lesquels sont aussi des abus d'oubli. $\gg^{5}$

La caractérisation concrète de ces abus de la mémoire comporte deux grands traits. Ils s'appuient, tout d'abord, sur la dimension narrative des phénomènes de

1. Jean-Michel Chaumont, "Connaissance ou reconnaissance ? Enjeux du débat sur la singularité de La shoah », art. cité, p. 79.

2. Ibid., p. 29.

3. Paul Ricœur, op. cit., p. 68.

4. « (La catégorie wébérienne de rationalité selon une fin - Zweckrationalität - opposée à celle de rationalité selon une valeur - Wertrationalität - est ici à sa place ; de même celle mise en œuvre par Habermas de "raison stratégique" opposée à "raison communicationnelle") 》 Il convient de remarquer la dimension purement et froidement calculatrice qui est sous-entendue par le choix d'une rationalité selon une fin et non selon une valeur.

5. Paul Ricœur, op. cit., p. 97. 
mémoire. «L'idéologisation de la mémoire est rendue possible par les ressources de variation qu' offre le travail de configuration narrative. ${ }^{1}$ Paul Ricœur insiste sur le rôle central qu'y joue la mise en récit : «Comme les personnages du récit sont mis en intrigue en même temps que l'histoire racontée, la configuration narrative contribue à modeler l'identité des protagonistes de l'action en même temps que les contours de l'action elle-même... C'est plus précisément la fonction sélective du récit qui offre à la manipulation l'occasion et les moyens d'une stratégie rusée qui consiste d'emblée en une stratégie de l'oubli autant que de la remémoration. $~^{2}$ Les abus de la mémoire prennent alors la forme d'une mise en récit qui fait ressortir certains personnages, à l'image de celle observée lors de l'étude de l'introduction de la figure du Juste au sein de la mémoire historique française de l'Occupation. De plus, à la vue de ses effets et implications, cette introduction peut apparaître comme s'insérant dans la poursuite d'objectifs précis. La figure du Juste peut donc être, par-là, rapprochée d'un pur instrument et son usage, d'une manipulation. Mise en récit et instrumentalisation d'une figure, cet usage de la mémoire revêtirait donc, à suivre Tzvetan Todorov, JeanMichel Chaumont ou Paul Ricœur, les traits d'un abus de la mémoire. L'étude de l'institutionnalisation de la figure du Juste pourrait donc fournir une illustration empirique au concept analytique central du paradigme de la mémoire stratégique.

Cependant, les abus de la mémoire se caractérisent, aussi et dans l'exact même temps, en ce qu'ils servent à affirmer une identité collective excluante, à définir le « nous » du groupe contre le « eux », hostile, des autres. En effet, les abus de la mémoire ne se conforment pas à « la vertu de justice par excellence et par constitution tournée vers autrui $»^{3}$. Or, l'usage de la figure du Juste constitue potentiellement un vecteur de rapprochement entre des groupes et des identités distincts comme celles de Juifs, de Français et de non-Juifs. Mais plus fondamentalement, derrière cette seconde caractéristique - et au cœur du recours à la notion d'abus de la mémoire - se trouve la question de l'horizon, la fin de la mémoire. En effet, Paul Ricœur considère avec intérêt le seul moyen d'éviter ces abus, c'est-à-dire le «conseil tout à fait judicieux de Todorov, d'extraire des souvenirs traumatisants la valeur exemplaire qu'un retournement de la mémoire en projet peut seul rendre pertinente $\gg^{4}$. Ainsi, par opposition, les abus de la mémoire façonnent-ils une mémoire tournée exclusivement vers le passé et le traumatisme, excluant certains au profit d'autres. Ils oblitèrent alors « la visée du futur, la question de la fin, de l'enjeu moral $\gg^{5}$ qui est, pour ces auteurs, au cœur de la mémoire. Or, manifestation empirique que ces auteurs n'étudient pas, la mise en scène de la figure du Juste met précisément en avant cette exemplarité de la mémoire et les valeurs d'héroïsme et de courage. Abus de la mémoire, car «manipulation » et mise en récit, l'institutionnalisation de la figure du Juste au sein la mémoire historique est, simultanément, une expression de cette mémoire exemplaire. Ainsi, son étude révèle-t-elle la présence d'une contradiction logique fondamentale au cœur du concept d'abus de mémoire, pierre angulaire des discours des tenants du paradigme de la mémoire stratégique.

Cette impasse s'explique notamment par le fait que les processus de constitution de ces «mémoires exemplaires » ne sont jamais étudiés pour eux-mêmes. Ainsi, celles-ci ne sont-elles définies que par leurs fins alors que les abus sont, eux, identifiés

1. Ibid., p. 103 .

2. Ibid.

3. Paul Riccur, op. cit., p. 108.

4. Ibid., p. 105.

5. Ibid. 


\section{Sarah Gensburger}

et décrits tant par leurs fins que par leurs moyens. Les variations entre les analyses successives de Jean-Michel Chaumont est ici révélatrice. En effet, « après le temps de la honte, vint celui d'une glorification dont un des effets les plus tangibles sera une fulgurante ascension statutaire des rescapés de la Shoah. Cette évolution - révolution serait un terme plus adéquat - ne s'est pas opérée toute seule en vertu d'on ne sait quel retour de balancier mécanique. Au contraire, elle a été activement poursuivie, et même si l'activisme n'explique pas tout, il donna l'impulsion du mouvement et en fut la condition nécessaire $\gg^{1}$. À l'inverse, lorsque, au sein du premier moment, exemplaire, de la mémoire, la mémoire des résistants occupait la première place, il ne faut pas «y voir l'effet d'une conspiration quelconque pour marginaliser les rescapés de la Shoah. Cette marginalisation a bien eu lieu, aussi bien au niveau des personnes que de l'événement, mais elle s'explique largement par des facteurs involontaires $\gg^{2}$.

Pour être exemplaire, la mémoire doit donc être perçue comme spontanée. Inversement, la mise en évidence empirique de la coexistence d'usages et d'exemplarité au sein d'une seule et même manifestation mémorielle est incompatible avec la prétention à utiliser la notion d'abus de la mémoire pour mener une analyse scientifique des phénomènes de mémoire ${ }^{3}$. L'épaisseur des mécanismes mémoriels n'est ici pas prise en considération. L'observation de la composition du « corpus » de travail de JeanMichel Chaumont est un indice supplémentaire de l'artificialité de cette approche de la mémoire. Ainsi sommes-nous tentée de suivre Pierre Bouretz lorsqu'il considère que l'« on peut avoir le sentiment qu'il fabrique le corpus avec quelques textes épars pour en faire les indices d'un projet collectif $»^{4}$. En effet, « ici Jean-Michel installe sa thèse sur un matériau épars trop rapidement érigé en corpus. Pour l'essentiel, en effet, tout repose sur deux ensembles : la discussion qui réunit Emil Fackenheim, Richard Pop, George Steiner et Elie Wiesel à New York en 1967 sur l'invitation de la revue Judaism, puis les travaux de Steven Katz déjà évoqués. Puis, de manière plus diffuse mais récurrente, les propos de Simone Veil ou Elie Wiesel $\gg^{5}$.

En effet, les causes et les acteurs de ces évocations du passé sont simplement déduits, de façon quasi-linéaire, de leurs effets et des valeurs morales ${ }^{6}$ respectives que l'observateur leur accorde. Au sein de l'approche analytique des tenants du paradigme de la mémoire stratégique, le terme de « mémoire » est donc utilisé dans un sens éminemment réducteur. Il ne fait référence qu'à un écran opaque et nulle attention n'est accordée aux différentes sources lumineuses qui, de par leur combinaison, sont pourtant à l'origine de l'image finale que celui-ci figure. La contradiction interne au concept d'abus de mémoire conduit donc à la remise en cause de la pertinence des outils analytiques utilisés par les tenants du paradigme de la mémoire stratégique et révèle leur méconnaissance des mécanismes empiriques de constitution de la mémoire considérée dans son épaisseur.

1. Jean-Michel Chaumont, op. cit., p. 93.

2. Ibid., p. 16.

3. Bien sûr, elles conservent leur efficacité idéologique pour dénoncer certaines mémoires et en soutenir d'autres. Mais tel n'est pas l'objet de la sociologie de la mémoire. p. 157.

4. Pierre Bouretz, « Cette fumée-ci, ils ne savent pas... », Le débat, janvier-février 1998,

5. Ibid., p. 162.

6. Il est d'ailleurs regrettable que les critiques de ces développements se sont jusqu'ici, elles aussi, situées principalement sur le terrain de la morale. Cf. notamment les articles de Pierre Bouretz, Richard Marientas, Michel Wieviorka et Jean-Michel Chaumont, dans Le débat, janvier-février 1998, qui rendent compte de certains aspects des questions soulevées par ce discours. 
Comment, dès lors, les phénomènes de remémoration doivent-ils être appréhendés ? Si la perception de la mémoire comme résultant $d^{\prime}$ une manipulation froide ${ }^{1}$ et organisée est apparue comme inopérante, doit-on pour autant penser la mémoire historique comme un reflet spontané d'un passé effectivement vécu, porté par des souvenirs individuels? « Peut-on distinguer vraiment d'une part une mémoire sans cadres, ou qui ne disposerait pour classer ses souvenirs que des mots du langage et de quelques notions empruntées à la vie pratique, d'autre part un cadre historique ou collectif, sans mémoire, c'est-à-dire qui ne serait point construit, reconstruit et conservé dans les mémoires individuelles ? Nous ne le croyons pas. ${ }^{2}$ En effet, de nombreux terrains d'étude ont confirmé l'intuition d'Halbwachs. Comme l'a souligné MarieClaire Lavabre ${ }^{3}$, suite à son étude de la mémoire communiste, l'institution d'une mémoire historique ne peut être opérante que si elle s'appuie sur une mémoire vive. En effet, se pose « la question de l'adéquation entre les images du passé dans la mémoire historique et celles que se forgent et véhiculent les individus $»^{4}$. La mémoire, telle qu'elle apparaît au niveau officiel, ne peut s'expliquer par des liens de causalité univoques, contrairement à ce que pourrait laisser entendre l'approche des tenants du paradigme de la mémoire stratégique. Elle appelle l'analyse simultanée de trois niveaux de mémoire qui sont en interactions complexes ${ }^{5}$ : la mémoire historique, norme mémorielle officielle, orientée vers l'homogénéisation de la collectivité et qui repose sur des usages politiques du passé ; la mémoire individuelle, souvenirs des individus qui apparaissent comme personnels ; la mémoire collective, qui se situe au croisement des deux précédentes en ce qu'elle lie identité, appartenance et rapport au passé. La mémoire individuelle apparaît alors comme un point de vue sur des mémoires collectives. Cependant, ces différents niveaux de mémoire ne doivent pas être réifiés. Idéaux-types et outils de travail, ils n'existent qu'entremêlés. Cette imbrication se retrouve au sein du processus d'institutionnalisation de la figure du Juste. Si celui-ci peut être présenté à travers la seule focale de la mémoire historique, cela ne suffit pas à comprendre les mécanismes concrets qui le portent.

En effet, si l'institutionnalisation de la figure du Juste résulte d'un usage officiel, elle n'en découle pas linéairement. Cet usage a, par exemple, dû s'infléchir suite à son interaction avec la force de mémoire que porte 1'Institut Yad Vashem. Mais, il apparaît, plus largement, que ce niveau historique de la mémoire des Justes ne se constitue qu'en s'articulant avec les autres forces de mémoire que constituent les mémoires vives, institutionnelles et collectives. Les entretiens que nous avons eu 1'occasion de réaliser montrent par exemple qu'il existe une articulation ${ }^{6}$ véritable entre mémoire vive et utilisation du souvenir des Justes par la mémoire historique. Tout d'abord, à travers la procédure d'attribution du titre de « Juste parmi les nations », des individus

1. Cf. note 4, p. 316. Le choix du concept de rationalité selon une fin, préféré à celui de rationalité en valeurs, est un exemple très fort du calcul froid associé à l'idée d'une manipulation.

2. Maurice Halbwachs, op. cit., p. 108.

3. Marie-Claire Lavabre, op. cit.

4. Marie-Claire Lavabre, op. cit., p. 36.

5. Pour une présentation de détail cf.: Marie-Claire Lavabre, «Peut-on agir sur la mémoire? », La mémoire entre histoire et politique, Cahiers français, 303, juillet-août 2001, p. 8-13.

6. Articulation dont - dans le cadre, par essence restreint, de cet article - nous n'avons pu rendre compte et dont la complexité et l'intérêt mériteraient de nombreuses pages. 


\section{Sarah Gensburger}

nourrissent de leurs souvenirs cette figure mémorielle ${ }^{1}$. Or, depuis 1995 , l'effectif des demandes d'attribution par les personnes sauvées a atteint un niveau élevé et s'y maintient. De même, dans les deux tiers des entretiens menés auprès des témoins, ceux-ci évoquent, eux aussi, spontanément les statistiques de survie utilisées par les mises en récit de la mémoire historique. Enfin, la mention, par les témoins, des actes de Résistance $^{2}$ des sauveteurs est très fréquente, tant dans les dossiers que dans les entretiens. D'ailleurs, la proximité entre les deux figures transparaît, plus largement, dans les entretiens menés avec les témoins. L'analyse de la reformulation de la mémoire historique de l'Occupation, sous les traits de la figure du Juste, appelle donc une prise en compte de l'ensemble des forces qui façonnent la mémoire des Justes ${ }^{3}$.

Le régime de la mémoire historique française de l'Occupation entre donc dans une nouvelle période ${ }^{4}$. En effet, celui-ci s'est trouvé renouvelé par l'institutionnalisation progressive du nouveau visage de la mémoire qu'incarne la figure du Juste parmi les nations. Des commémorations, des discours et des textes officiels ont, depuis 1995 , marqué les différentes étapes de ce processus. Ce dernier s'est déroulé selon des modalités diverses. Mais en leur sein, la relation établie entre figure du Juste et figure du Résistant occupe une place centrale. D'une part, la première s'insère dans la mémoire historique nationale en s'appuyant sur les ressorts symboliques qui ont structuré la seconde. De l'autre, la figure du Juste occupe un rôle fonctionnel, au sein d'une rhétorique qui demeure dichotomique, autrefois dévolu aux seuls résistants.

La mise en place de ce nouveau régime de mémoire historique tend vers la réconciliation de la nation avec elle-même. Elle structure une mise en récit du passé qui réconcilie Juifs et non-Juifs, mais aussi mémoires des déportations tant « politique » que « raciale ». La portée réconciliatrice s'appuie cependant sur une répétition systématique des statistiques de sauvetage des Juifs de France. Elle met en évidence l'importance de l'imbrication des figures collectives et individuelles au sein des phénomènes de mémoire. Ce régime de mémoire permet notamment le glissement du titre israélien initial de «Juste parmi les nations» vers un titre réapproprié de «Juste de France » qui, au-delà de lui-même, incarne la société civile française dans sa globalité.

1. Au moins 6000 Juifs peuvent être considérés comme ayant initié cette mise en avant de non-Juifs «exemplaires » et qu'ils considèrent comme leurs amis, voire leurs familles. Ce nombre ne doit pas être mis en perspective avec l'ensemble des Juifs de France car seules les personnes directement témoins des faits peuvent témoigner. Le sauvetage ayant très souvent concerné des enfants, les parents de l'époque (c'est-à-dire les plus de 80 ans) mais surtout les Juifs nés après la guerre (les 1-55 ans) ne peuvent témoigner. $D^{\prime}$ ailleurs, les entretiens montrent que ces derniers soutiennent, presque systématiquement, et souvent par un investissement personnel concret, la démarche de leurs parents qui ouvrent un dossier auprès du Comité français pour Yad Vashem.

2. Au sens traditionnel de la résistance armée.

3. Dans le corps du présent article, nous avons choisi de présenter la figure du Juste à travers la focale de la mémoire historique. Cepèndant, ce texte s'insère au sein d'un travail plus large qui cherche à comprendre la «mémoire des Justes » dans l'ensemble de ses composantes. L'isolement d'un seul niveau de mémoire ne doit pas être confondu avec une quelconque réification, il ne répond qu'à un souci de méthode. Les développements qui viennent d'être faits ne donnent donc qu'un aperçu de phénomènes beaucoup plus larges et complexes.

4. Cf. la réflexion comparative de Maurice Kriegel, «Trois mémoires de la Shoah : ÉtatsUnis, Israèl, France. À propos de Peter Novick, L'Holocauste dans la vie américaine », Le débat, 117, novembre-décembre 2001, p. 59-73. 


\section{Les figures du «Juste » dans la mémoire de l'occupation}

C'est donc à ce Juste nationalisé que la loi du 23 mars 2000 fait référence en instituant, à la date du 16 juillet, « une journée nationale à la mémoire des victimes des crimes racistes et antisémites de l'État français et d'hommage aux "Justes" de France ».

La succession des différentes étapes de cette rappropriation d'une figure par la mémoire historique donne l'impression d'une «manipulation », voire de ce que les tenants du paradigme de la mémoire stratégique qualifient d' « abus de la mémoire ». Pourtant, la confrontation de ce dernier concept à la réalité empirique de l'institutionnalisation de la figure du Juste révèle le caractère inopérant d'une telle approche. Elle oblige à une réflexion méthodologique et conceptuelle sur la notion de mémoire. Les interactions entre différents niveaux de mémoire, qui sont autant d'outils de recherche, apparaissent comme des terrains privilégiés d'approche des mécanismes de mémoire. Ainsi, l'approche linéaire, unidimensionnelle et purement stratégique de la mémoire se révèle inopérante «tant il est vrai que les cadres collectifs de la mémoire ne se ramènent pas à des dates, à des noms et à des formules, qu'ils représentent des courants de pensée et d'expérience où nous retrouvons notre passé parce qu'il en a été traversé ${ }^{1 *}$.

Sarah Gensburger, diplômée de l'Institut d'études politiques de Paris et ancienne élève de l'École normale supérieure de Cachan, est doctorante à l'École des hautes études en sciences sociales, rattachée au Centre interdisciplinaire d'étude des faits religieux. Sa thèse en cours porte sur la mémoire des Justes parmi les nations en France. Elle y étudie cette mémoire dans l'ensemble de ses dimensions tant individuelle, collective qu'historique. Elle est l'auteur de : «Le cadre institutionnel de la « mémoire des Justes » : Figures, témoignages et souvenirs », mémoire de DEA de sociologie, sous la direction de Marie-Claire Lavabre, Paris, EHESS, 2000 ; «Identité sociale et situation extrême : le cas des camps de concentration », Documents pour l'enseignement économique et social, mars 1998. Agrégée de sciences économiques et sociales, elle enseigne la sociologie en $1^{\text {er }}$ cycle, à $1^{\prime}$ Université Paris VIII-Saint-Denis. (18 rue de Mazagran 75010 Paris, <sgensburger@yahoo.fr>)

\section{RÉSUMÉ/ABSTRACT}

LES FIGURES DU JUSTE ET DU RÉSISTANT ET L'ÉVOLUTION DE LA MÉMOIRE HISTORIQUE FRANÇAISE DE L'OCCUPATION

L'article propose de renouveler l'analyse de la mémoire historique française de l'Occupation. Il met en évidence un processus d'institutionnalisation d'une nouvelle figure, celle du Juste parmi les nations devenu progressivement Juste de France. Cette évolution repose notamment sur un rapport complexe entre ce nouveau personnage mémoriel et celui, plus ancien, du Résistant. D'une part, cette mise en place s'appuie sur des modalités symboliques proches de celles

1. Maurice Halbwachs, op. cit., p. 113.

* Cet article s'appuie sur une recherche réalisée dans le cadre d'une maîtrise de sociologie réalisée en 1998, à Paris X-Nanterre-ENS Cachan, intitulée «Forces et enjeux de mémoire en France : la figure du Juste », sous la direction de Marc Lazar, et dans le cadre du DEA de sociologie à l'École des hautes études en sciences sociales, intitulé «Le cadre institutionnel de la "mémoire des Justes" : Figures, témoignages et souvenirs », sous la direction de Marie-Claire Lavabre. Je tiens à exprimer ma très grande gratitude à Marie-Claire Lavabre pour ses minutieuses relectures et ses précieux conseils. 
utilisées pour les résistants. D'autre part, la figure du Juste de France remplit un rôle fonctionnel autrefois exclusivement dévolu à ces derniers. À travers son incorporation s'opère la réécriture d'une rhétorique dichotomique au sein d'un régime mémoriel renouvelé où une France-patrie des droits de l'homme remplace une France-nation et territoire. Ce constat conduit à l'analyse de la portée réconciliatrice d'une telle mémoire historique et de ses modalités. Celle-ci repose notamment sur les imbrications entre individuel et collectif au cour des représentations du passé. L'article incite enfin à un retour critique sur les travaux des tenants $d u$ " paradigme de la mémoire stratégique ». Ainsi, à travers un cas empirique concret, l'auteur se livre-t-il à une réflexion conceptuelle et méthodologique sur la notion de mémoire et ses implications. Apparaît avec force la nécessité de dépasser les approches courantes des phénomènes de mémoire, souvent trop linéaires et unidimensionnelles.

\section{THE «RIGHTEOUS» AND RESISTANT CHARACTERS IN THE FRENCH HISTORIC MEMORY OF THE OCCUPATION}

The article aims to throw new light on the analysis of French historical memory of the occupation. There is the institutionalization of a new figure, that of the righteous among nations becoming progressively the righteous of France. This development is based on complex relations between this new memorial character and the older one of the Resistant. On the one hand, this character is built on symbolic processes similar to those used for the Resistants, and on the other hand, the figure of the righteous of France fulfills a functional role that previously fell only to the latter. It is incorporated through the rewriting of a dichotomic rhetoric within a renewed memorial regime in which a France - land of Human Rights replaces a France-Nation and Territory. This observation leads to the analysis of the reconciliatory scope of such a historic memory and its methods. It is based in particular on the intertwining between the individual and the group within the representations of the past. It fosters a critical review of the work of those who defend the " paradigm of strategic memory ». Thus, through a concrete empiric case, the author deals with a conceptual and methodological thinking on the notion of memory and its implications. It is essential to go beyond the common approaches of the phenomena of memory, all too often linear and one-dimensional. 